\title{
Does black-hole growth depend on the cosmic environment?
}

\author{
G. Yang(杨光), ${ }^{1,2 \star ~ W . ~ N . ~ B r a n d t, ~}{ }^{1,2,3}$ B. Darvish, ${ }^{4}$ C.-T. J. Chen(陳建廷), ${ }^{1,2}$ F. Vito, ${ }^{1,2}$ \\ D. M. Alexander, ${ }^{5}$ F. E. Bauer ${ }^{6,7,8}$ and J. R. Trump ${ }^{9}$ \\ ${ }^{1}$ Department of Astronomy and Astrophysics, 525 Davey Lab, The Pennsylvania State University, University Park, PA 16802, USA \\ ${ }^{2}$ Institute for Gravitation and the Cosmos, The Pennsylvania State University, University Park, PA 16802, USA \\ ${ }^{3}$ Department of Physics, 104 Davey Laboratory, The Pennsylvania State University, University Park, PA 16802, USA \\ ${ }^{4}$ Cahill Center for Astrophysics, California Institute of Technology, 1216 East California Boulevard, Pasadena, CA 91125, USA \\ ${ }^{5}$ Centre for Extragalactic Astronomy, Department of Physics, Durham University, South Road, Durham DH1 3LE, UK \\ ${ }^{6}$ Instituto de Astrofísica and Centro de Astroingeniería, Facultad de Física, Pontificia Universidad Católica de Chile, Casilla 306, Santiago 22, Chile \\ ${ }^{7}$ Millennium Institute of Astrophysics (MAS), Nuncio Monseñor Sótero Sanz 100, Providencia, Santiago, Chile \\ ${ }^{8}$ Space Science Institute, 4750 Walnut Street, Suite 205, Boulder, CO 80301, USA \\ ${ }^{9}$ Department of Physics, University of Connecticut, 2152 Hillside Road, U-3046, Storrs, CT 06269, USA
}

Accepted 2018 July 14. Received 2018 June 27; in original form 2018 May 1

\begin{abstract}
It is well known that environment affects galaxy evolution, which is broadly related to supermassive black hole (SMBH) growth. We investigate whether SMBH evolution also depends on host-galaxy local (sub-Mpc) and global $(\approx 1-10 \mathrm{Mpc})$ environment. We construct the surface-density field (local environment) and cosmic web (global environment) in the Cosmic Evolution Survey (COSMOS) field at $z=0.3-3.0$. The environments in COSMOS range from the field to clusters $\left(M_{\text {halo }} \lesssim 10^{14} \mathrm{M}_{\odot}\right.$ ), covering the environments where $\approx 99$ per cent of galaxies in the Universe reside. We measure sample-averaged SMBH accretion rate ( $\overline{\mathrm{BHAR}})$ from X-ray observations, and study its dependence on overdensity and cosmic-web environment at different redshifts while controlling for galaxy stellar mass $\left(M_{\star}\right)$. Our results show that $\overline{\text { BHAR }}$ does not significantly depend on overdensity or cosmic-web environment once $M_{\star}$ is controlled, indicating that environment-related physical mechanisms (e.g. tidal interaction and ram-pressure stripping) might not significantly affect SMBH growth. We find that $\overline{\mathrm{BHAR}}$ is strongly related to host-galaxy $M_{\star}$, regardless of environment.
\end{abstract}

Key words: galaxies: active-galaxies: evolution-galaxies: nuclei-large-scale structure of Universe-X-rays: galaxies.

\section{INTRODUCTION}

The environments of galaxies play a crucial role in their evolution (e.g. De Lucia et al. 2006; Conselice 2014; Somerville \& Davé 2015). In the local Universe, denser regions are preferentially populated by early-type quiescent galaxies, while less-dense regions are more likely to host late-type star-forming galaxies (e.g. Dressler 1980; Balogh et al. 2004; Kauffmann et al. 2004). This environmental dependence of star-forming/quiescent types exists at $z \lesssim 1$, although it is less clear at higher redshifts (e.g. Cooper et al. 2006; Elbaz et al. 2007; Peng et al. 2010; Scoville et al. 2013; Darvish et al. 2016).

Several possible environment-related mechanisms could affect galaxy evolution. Cold gas, the fuel of star formation, could flow into galaxies through cosmic filaments (e.g. Kereš et al. 2005; Dekel et al. 2009); frequent tidal interactions in denser regions could

\footnotetext{
^E-mail: gxy909@psu.edu
}

effectively deplete cold gas (e.g. Farouki \& Shapiro 1981; Moore, Lake \& Katz 1998); the strong ram pressure in clusters can strip cold gas from galaxies and suppress subsequent star formation (e.g. Gunn \& Gott 1972; Ebeling, Stephenson \& Edge 2014; Poggianti et al. 2016); and mergers, which can fundamentally change galaxy properties, happen more frequently in high-density regions (e.g. Hopkins et al. 2006; Lin et al. 2010). These physical processes might also affect active galactic nucleus (AGN) activity, as the growth of supermassive black holes (SMBHs) also relies on the supply of cold gas (e.g. Alexander \& Hickox 2012; Vito et al. 2014; Poggianti et al. 2017).

Optical observations of low-redshift $(z \lesssim 1)$ quasars disagree on whether they tend to reside in high- or low-density regions compared to normal galaxies (e.g. Serber et al. 2006; Strand, Brunner \& Myers 2008; Lietzen et al. 2009). This disagreement might be caused if these works did not carefully control for host-galaxy properties. Karhunen et al. (2014) found quasars do not show a significant dependence on environment compared to normal galaxies with matched redshift and host-galaxy luminosities. At high redshift 
( $z \gtrsim 3$ ), optical observations are limited to rare luminous quasars, and deep spectroscopic observations are often needed to measure their environment. Therefore, these studies are often limited to small sample sizes and statistically significant conclusions cannot be obtained (e.g. Bañados et al. 2013; Overzier 2016; Balmaverde et al. 2017).

Optical selection is often biased to luminous broad-line (BL) quasars, especially at high redshift. These BL quasars are rare and not well representative of the whole AGN population. X-ray emission can trace AGN activity down to a modest level and is widely used to investigate SMBH growth over the majority of cosmic history (e.g. Brandt \& Alexander 2015; Xue 2017). Studies of AGN activity versus environment found that, at low redshift $(z \lesssim 1)$, the $\mathrm{X}$-ray AGN fraction in rare rich clusters is generally lower than that in the field (e.g. Martini, Sivakoff \& Mulchaey 2009; Ehlert et al. 2014; but also see e.g. Haggard et al. 2010). At higher redshifts, relevant studies are often constrained to rare protoclusters with limited AGN/galaxy sample sizes. Their results suggest that AGN activity tends to be enhanced in these protoclusters (e.g. Lehmer et al. 2009; Digby-North et al. 2010; Lehmer et al. 2013; Martini et al. 2013; Umehata et al. 2015; Alexander et al. 2016; but also see Macuga et al. 2018).

However, this apparent environmental dependence might only be a secondary effect, and SMBH growth might be more fundamentally related to host-galaxy properties which are themselves related to environment. For example, $\mathrm{X}$-ray AGN activity is strongly related to host-galaxy stellar mass $\left(M_{\star}\right)$ rather than colour (e.g. Xue et al. 2010) or star formation rate (SFR; e.g. Yang et al. 2017), and thus $M_{\star}$ must be carefully controlled when assessing AGN dependence on other host-galaxy properties. On the other hand, massive galaxies tend to reside in high-density regions (e.g. Coil et al. 2006, 2017). Therefore, to avoid such $M_{\star}$-related biases, a large sample of AGNs and galaxies is needed to investigate the accretion-environment relation, while controlling for host-galaxy $M_{\star}$.

In this paper, we study the dependence of sample-averaged SMBH accretion rate ( $\overline{\mathrm{BHAR}})$ on galaxy overdensity and cosmicweb environment while controlling for $M_{\star}$. The sample-averaged SMBH accretion is employed to approximate long-term average SMBH accretion for a galaxy sample (e.g. Chen et al. 2013; Hickox et al. 2014; Yang et al. 2017, 2018), because AGNs plausibly have strong variability on timescales of $\sim 10^{2}-10^{7} \mathrm{yr}$ (e.g. Martini \& Schneider 2003; Novak, Ostriker \& Ciotti 2011; Sartori et al. 2018). Here, we define the overdensity as the galaxy surface number density relative to the median value at a given redshift and cosmic-web environment as a galaxy's association to the field, a filament, or a cluster. The overdensity and cosmic-web environment are assessed on physical scales of sub-Mpc and $\approx 1-10 \mathrm{Mpc}$, respectively. Hereafter, we refer to overdensity and cosmic-web environment as 'local' and 'global' environments, respectively.

Our aim is to probe a wide redshift range of $z=0.3-3.0$ with large samples of X-ray AGNs $(\approx 2000)$ and galaxies $(\approx 170000)$. In particular, this range covers $z \approx 1.5-2.5$, the peak of cosmic AGN and star formation activity, when various physical processes such as galaxy mergers and AGN feedback likely play an important role in shaping SMBH and galaxy co-evolution (e.g. Conselice 2014; Madau \& Dickinson 2014; Brandt \& Alexander 2015; King \& Pounds 2015).

Our analyses are based on the Cosmic Evolution Survey (COSMOS, e.g. Scoville et al. 2007; McCracken et al. 2012). COSMOS has been intensively covered by spectroscopic and multiwavelength imaging observations (e.g. Lilly et al. 2009; Laigle et al. 2016).
Over 20000 sources have secure spectroscopic redshifts (spec-z), while other sources have reliable photometric redshifts (photo- $z$ ) derived from high-quality ultraviolet-to-infrared (UV-to-IR) data (up to 32 bands; e.g. Laigle et al. 2016). The UV-to-IR data also make it possible to assess host-galaxy properties such as $M_{\star}$ and starforming/quiescent type (e.g. Ilbert et al. 2013; Davidzon et al. 2017). Deep Chandra X-ray observations ( $\approx 160$ ks exposure), which can be used to measure SMBH growth, are also available from the COSMOS-Legacy survey (Civano et al. 2016). The excellent X-ray positions from Chandra $(\approx 0.5 \mathrm{arcsec})$ enables reliable matching between X-ray and optical sources (Marchesi et al. 2016a).

Thanks to its relatively large area $\left(\approx 2 \mathrm{deg}^{2}\right)$ and deep panchromatic coverage, COSMOS is one of the major fields for environment studies. State-of-the-art techniques have been applied to COSMOS to derive reliable measurements of the surface-density field up to $z \approx 3$ (e.g. Scoville et al. 2013; Darvish et al. 2015). The statistical properties of the resulting density field such as mean densities and density ranges agree with the predictions from cosmological simulations (e.g. Scoville et al. 2013). Based on the density field, Darvish et al. (2017) utilized a new technique to construct a measurement of the cosmic web (Aragón-Calvo et al. 2007). This method allows the mapping of sources to clusters, filaments, and the field.

This paper is structured as follows. In Section 2, we describe our data analyses. In Section 3, we present our results. We discuss our results in Section 4 and summarize our study in Section 5.

Throughout this paper, we assume a cosmology with $H_{0}=70 \mathrm{~km}$ $\mathrm{s}^{-1} \mathrm{Mpc}^{-1}, \Omega_{\mathrm{M}}=0.3$, and $\Omega_{\Lambda}=0.7$, and a Chabrier initial mass function (Chabrier 2003). Quoted uncertainties are at the $1 \sigma$ (68 per cent) confidence level, unless otherwise stated. We express $M_{\star}, M_{\mathrm{BH}}$, and $M_{\text {halo }}$ (halo mass) in units of $\mathrm{M}_{\odot}$ and $\overline{\mathrm{BHAR}}$ in units of $\mathrm{M}_{\odot} \mathrm{yr}^{-1} . L_{\mathrm{X}}$ indicates AGN X-ray luminosity at rest-frame $2-10 \mathrm{keV}$ and is in units of erg s $\mathrm{s}^{-1}$. All lengths/distances are in physical (proper) scale, unless otherwise stated.

\section{DATA ANALYSES}

\subsection{Galaxy sample selection}

Our data are based on the COSMOS2015 survey (Laigle et al. 2016). We only utilize sources within both the COSMOS and UltraVISTA regions, and remove objects in masked regions (e.g. bad pixels in detectors). These sources cover an area of $\approx 1.4 \mathrm{deg}^{2}$ (see fig. 1 and table 7 in Laigle et al. 2016). The UltraVISTA region has deep NIR imaging data that are essential in estimating photo- $z$ and $M_{\star}$ (Section 2.2). We restrict our study to the $\approx 170000$ sources brighter than $K_{\mathrm{S}}=24$ (the $3 \sigma$ limiting magnitude of the COSMOS2015 catalogue) to avoid large uncertainties of photo- $z$ for faint sources. The basic properties of our sample are listed in Table 1. Our analyses (Section 3$)$ are performed for the three redshift bins $(z=0.3-1.2$, 1.2-2, and 2-3) listed in Table 1. These redshift bins cover comoving volumes of $7 \times 10^{6}, 1.2 \times 10^{7}$, and $1.7 \times 10^{7} \mathrm{Mpc}^{3}$, respectively. Table 2 shows a portion of our source catalogues, and the full version is available as Supporting Information.

We obtain spec- $z$ for $\approx 20000$ sources in our sample (see Table 1; Marchesi et al. 2016a; Delvecchio et al. 2017; Salvato et al. in preparation). ${ }^{1}$ For sources without spec- $z$, we adopt the photo$z$ measurements from the COSMOS2015 catalogue. These mea-

\footnotetext{
${ }^{1}$ In the late stages of this work, a new spec- $z$ data set, the DEIMOS $10 \mathrm{k}$ catalogue, was released (Hasinger et al. 2018). This catalogue could increase our spec- $z$ sample by $\approx 10$ per cent, unlikely to affect our qualitative results.
} 
Table 1. Summary of sample properties.

\begin{tabular}{|c|c|c|c|c|c|c|c|c|c|c|c|}
\hline $\begin{array}{l}\text { Redshift } \\
\text { (1) }\end{array}$ & $\begin{array}{c}N_{\text {gal }} / N_{\text {spec }} \\
\text { (2) }\end{array}$ & $\begin{array}{c}\sigma_{\mathrm{NMAD}} \\
(3)\end{array}$ & $\begin{array}{c}\eta(\%) \\
(4)\end{array}$ & $\begin{array}{c}\log M_{\star, \text { med }} \\
\quad(5)\end{array}$ & $\begin{array}{c}\text { Frac }_{Q}(\%) \\
(6)\end{array}$ & $\begin{array}{l}N_{\text {slice }} \\
\text { (7) }\end{array}$ & $\begin{array}{c}\log (1+\delta) \\
(8)\end{array}$ & $\begin{array}{c}N_{\text {field }} / N_{\text {fila }} / N_{\text {clu }} \\
\text { (9) }\end{array}$ & $\begin{array}{l}N_{\mathrm{X}} \\
(10)\end{array}$ & $\begin{array}{c}E_{\text {rest }}(\mathrm{keV}) \\
(11)\end{array}$ & $\begin{array}{c}\log L_{X} \\
(12)\end{array}$ \\
\hline $0.3-1.2$ & $94152 / 18099$ & 0.011 & 2 & 9.3 & 13 & 180 & $(-0.16,0.18)$ & $38840 / 48960 / 6352$ & 889 & $(0.9,12.5)$ & $(42.6,43.3)$ \\
\hline $1.2-2.0$ & $48981 / 1322$ & 0.020 & 3 & 9.8 & 7 & 80 & $(-0.13,0.13)$ & $20365 / 28616 /-$ & 701 & $(1.3,17.7)$ & $(43.3,43.9)$ \\
\hline $2.0-3.0$ & $22828 / 412$ & 0.059 & 10 & 9.9 & 2 & 50 & $(-0.14,0.13)$ & $14265 / 8563 /-$ & 429 & $(1.7,23.6)$ & $(43.7,44.2)$ \\
\hline
\end{tabular}

Notes. (1) Redshift bins. (2) Numbers of galaxies and spec- $z$ sources in our sample $\left(K_{\mathrm{S}}<24\right)$. (3) Photo- $z$ uncertainty (compared to spec-z). (4) Photo- $z$ outlier fraction. (5) Median stellar mass. (6) Fraction of quiescent galaxies. (7) Number of $z$-slices. (8) The overdensity (25per cent, 75 per cent) percentile range. (9) Number sources in the field/filament/cluster environments. We do not assign cluster environment at $z>1.2$ due to its generally weak signals (see Section 2.3.2). (10) Number of X-ray-detected sources. (11) Rest-frame X-ray energy sampled at median redshift. (12) The (25per cent,75 per cent) percentile range of $\log L_{X}$ for X-ray-detected sources.

Table 2. Source catalogue.

\begin{tabular}{|c|c|c|c|c|c|c|c|c|c|c|}
\hline $\begin{array}{l}\text { RA } \\
\text { (1) }\end{array}$ & $\begin{array}{l}\text { Dec. } \\
\text { (2) }\end{array}$ & $\begin{array}{l}K_{\mathrm{S}} \\
(3)\end{array}$ & $\begin{array}{c}z \\
(4)\end{array}$ & $\begin{array}{l}z_{\text {lo }} \\
(5)\end{array}$ & $\begin{array}{l}z_{\text {up }} \\
(6)\end{array}$ & $\begin{array}{l}\log M_{\star} \\
\text { (7) }\end{array}$ & $\begin{array}{c}\text { Type }_{\text {gal }} \\
\text { (8) }\end{array}$ & $\begin{array}{c}\log (1+\delta) \\
(9)\end{array}$ & $\begin{array}{l}\text { Web } \\
(10)\end{array}$ & $\begin{array}{c}\log L_{X} \\
\text { (11) }\end{array}$ \\
\hline 149.411496 & 2.712315 & 22.8 & 0.706 & 0.655 & 0.774 & 9.18 & 1 & -0.343 & 2 & -99.00 \\
\hline 149.411576 & 2.336084 & 21.0 & 1.783 & 1.729 & 1.818 & 11.22 & 1 & 0.292 & 1 & -99.00 \\
\hline 149.411578 & 2.306681 & 21.3 & 1.359 & 1.241 & 1.433 & 10.71 & 0 & -0.019 & 1 & -99.00 \\
\hline 149.411581 & 2.411649 & 21.5 & 0.389 & 0.381 & 0.398 & 9.28 & 1 & 0.149 & 1 & -99.00 \\
\hline 149.411603 & 2.243533 & 21.9 & 1.502 & 1.469 & 1.543 & 10.32 & 1 & 0.285 & 1 & -99.00 \\
\hline 149.411659 & 2.319370 & 23.6 & 1.022 & 0.812 & 1.148 & 9.23 & 1 & -0.621 & 2 & -99.00 \\
\hline 149.411661 & 2.410365 & 22.5 & 1.063 & 1.063 & 1.063 & 9.11 & 1 & -0.192 & 1 & 43.63 \\
\hline
\end{tabular}

Notes. Only a portion of this table is shown here, and the full version is available as supplementary materials. The table is sorted in ascending order of RA. (1) and (2) Source J2000 coordinates. (3) $K_{\mathrm{S}} \mathrm{AB}$ magnitude from the COSMOS2015 catalogue (Laigle et al. 2016). (4)-(6) Redshift and redshift $1 \sigma$ lower and upper limits (Section 2.1). For spec- $z$ sources, the lower and upper limits are set the same as the redshift value. (7) Stellar mass (Section 2.2). (8) Galaxy type (0: quiescent; 1: star-forming; Section 2.2). (9) Overdensity (Section 2.3.1). (10) Cosmic-web environment (0: cluster; 1: filament; 2: field; Section 2.3.2). We do not assign cluster environment at $z>1.2$ due to its generally weak signals. (11) X-ray luminosity (rest-frame 2-10 keV; Section 2.4.1). For X-ray-undetected sources, the values are set to ' -99.00 '.

surements are derived from high-quality UV-to-NIR photometric data including 18 broad, 12 medium, and 2 narrow bands (see table 1 in Laigle et al. 2016). The medium bands can effectively improve the photo- $z$ quality, enabling reliable environment studies in COSMOS (e.g. Darvish et al. 2015; Darvish et al. 2017). When compared to different spec- $z$ catalogues, the photo- $z$ have $\sigma_{\mathrm{NMAD}} \approx 0.007-0.06$ and outlier $\left(|\Delta z| /\left(1+z_{\text {spec }}\right)>0.15\right)$ fraction $\eta \approx 0.5$ per cent -10 per cent (see table 5 in Laigle et al. 2016), where $\sigma_{\text {NMAD }}$ is defined as $1.48 \times \operatorname{median}\left(\frac{|\Delta z-\operatorname{median}(\Delta z)|}{1+z_{\text {spec }}}\right)$ (e.g. Yang et al. 2014). When compared with the recently released DEIMOS 10k spec- $z$ catalogue (Hasinger et al. 2018), the COSMOS2015 photo- $z$ have $\sigma_{\mathrm{NMAD}}=0.015$ and $\eta=8$ per cent, further demonstrating the high photo- $z$ quality of the COSMOS2015 catalogue. We consider all galaxies (including X-ray detected and undetected) when deriving $\overline{\mathrm{BHAR}}$ (see Section 2.4).

\subsection{Stellar mass}

To estimate $M_{\star}$, we perform spectral energy distribution (SED) fitting with CIGALE (Noll et al. 2009; Serra et al. 2011) at $z_{\text {spec }}$ or $z_{\text {photo }}$ (Section 2.1). The input photometry is from the COSMOS2015 catalogue (Section 2.1). We do not adopt the $M_{\star}$ measurements from the COSMOS2015 catalogue directly, mainly because the our redshifts are not exactly the same as those in the COSMOS2015 catalogue (Section 2.1). We employ nebular and dust emission in CIGALE (Noll et al. 2009; Draine \& Li 2007). We apply the extinction law from Calzetti et al. (2000) with $E(B-V)$ ranging from 0 to 1 . Following Yang et al. (2018), we use a $\tau$ model of the star formation history with $\log (\tau / \mathrm{yr})$ ranging from 8 to 10.5 . We allow stellar metallicity values of $Z=0.0001,0.0004,0.004,0.008,0.02$, and0.05, where
$Z$ is the mass fraction of metals. Our $M_{\star}$ measurements have a systematic of 0.002 dex and a scatter of 0.11 dex compared to those in the COSMOS2015 catalogue. For the 239 BL AGNs (identified by Marchesi et al. 2016a), we also adopt an additional BL AGN component following the settings in table 1 of Ciesla et al. (2015). The resulting $M_{\star}$ values are typically $\approx 0.3$ dex different from those obtained with only galaxy templates (see section 2.1.3 of Yang et al. 2018). Fig. 1 displays $M_{\star}$ versus redshift for our sample. We also show the $M_{\star}$ completeness limit corresponding to $K_{\mathrm{S}}=24$ from Laigle et al. (2016) in Fig. 1. The completeness limit is estimated based on an empirical method which does not assume a specific galaxy template. The limiting $\log M_{\star}$ at $z=1.2,2$, and 3 are 9.3, 10.0 , and 10.3, respectively. In Section 3, we perform analyses for $M_{\star}$ above these limits in three redshift bins of $z=0.3-1.2,1.2-2$, and $2-3$, respectively.

We classify a source as a quiescent galaxy if its rest-frame colours satisfy NUV $-r>3(r-J)+1$ and NUV $-r>3.1$, otherwise we classify it as a star-forming galaxy (Williams et al. 2009; Ilbert et al. 2010). ${ }^{2}$ Here, the rest-frame colours are obtained from our SED fitting. This colour-based selection helps to avoid misclassifying dust-reddened star-forming galaxies as quiescent galaxies (e.g. Ilbert et al. 2010, 2013). The fractions of quiescent galaxies in different redshift ranges are listed in Table 1. This classification is used to estimate X-ray emission from X-ray binaries (XRBs; see Section 2.4.3). Our colour-colour scheme is not appropriate for galaxies hosting BL AGNs due to the strong AGN UV-to-NIR emission. Following Yang et al. (2017), we set the hosts of BL AGNs as star-forming galaxies. Setting them as either star-forming

\footnotetext{
${ }^{2}$ Here, the NUV specifically refers to the GALEX band centred at $2300 \AA$.
} 


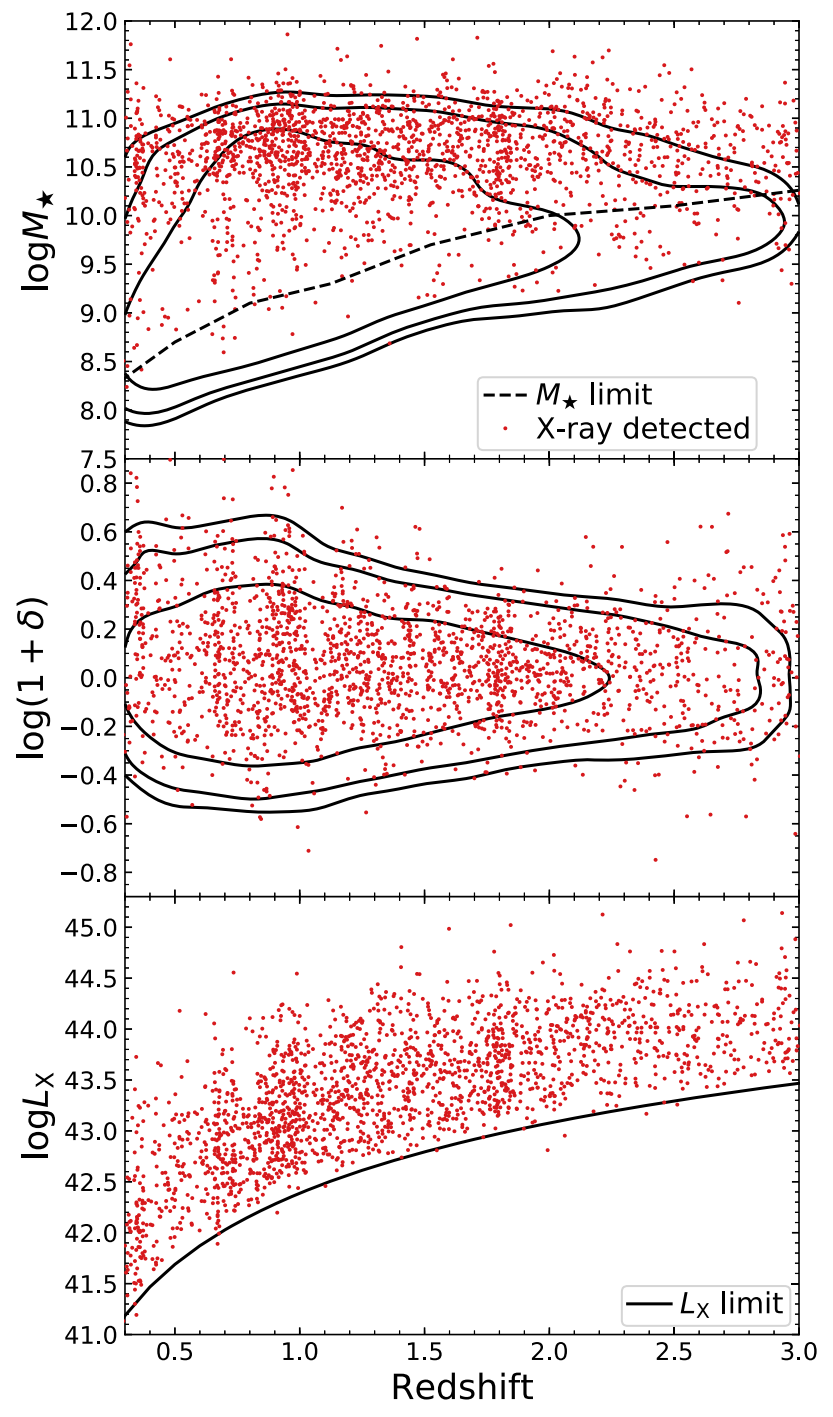

Figure 1. $M_{\star}$, overdensity, and $L_{\mathrm{X}}$ as a function of redshift. The contours encircle 68 per cent, 90 per cent, and 95 per cent of all sources (Section 2.1), respectively. The red points represent X-ray-detected sources. In the top panel, the dashed curve indicates the $M_{\star}$ completeness limit from Laigle et al. (2016). In the bottom panel, the solid curve represents the limiting $L_{X}$.

or quiescent galaxies has negligible effects to our results, as BL AGNs are only a small population compared to the entire galaxy sample $(\approx 0.1$ per cent $)$.

\subsection{Environment}

We build the surface-density field and cosmic-web estimates in this section. The technical details are presented in Darvish et al. (2015, 2017), and we briefly describe the procedures in Sections 2.3.1 and 2.3.2. In Appendix, we explain our environment measurements in a straightforward way, especially for readers who are not familiar with environmental studies. As demonstrated in Section 4.1, the physical environment-SFR relation clearly exists in our sample, supporting the robustness of our environment measurements.

Some studies suggest that there might be different environmental effects for 'central' versus 'satellite' galaxies in a dark-matter halo (e.g. Li et al. 2006; Hickox et al. 2009). We do not label our sources as central or satellite galaxies, because most galaxies $(\approx 80$ per cent -90 per cent $)$ at low redshift $(z \lesssim 0.6)$ are observed to be isolated or reside in small groups (galaxy members $\lesssim 5$ ) and their central/satellite classification is challenging due to factors like photo- $z$ uncertainties and survey sensitivity (e.g. Knobel et al. 2009, 2012). At higher redshift, the fraction of isolated or small-group galaxies is even higher as the large-scale structure is still in development (e.g. Springel et al. 2005; Overzier 2016). Considering that our sources cover a wide redshift range of $z=0.3-3$, a detailed unbiased central/satellite classification is beyond the scope of this work.

\subsubsection{Density field (local environment)}

We adopt the 'weighted adaptive kernel smoothing' method to construct the surface-density field that probes sub-Mpc physical scales. As demonstrated by intensive simulations, the performance of this method is excellent (see sections 5 and 6 of Darvish et al. 2015). The density field is calculated for all sources, including normal galaxies and X-ray-detected sources.

We first calculate $\sigma_{|\Delta z| /(1+z)}$ as a function of redshift. $\sigma_{|\Delta z| /(1+z)}$ is derived within $z \pm 0.2$ at each redshift. $\sigma_{|\Delta z| /(1+z)}$ is $\approx 0.01$ at low redshift $(z \lesssim 1)$ and rises to $\approx 0.04$ toward high redshift $(z \gtrsim 2)$. This level of photo- $z$ accuracy is sufficient for reliable cosmicenvironment characterization (e.g. Scoville et al. 2013; Darvish et al. 2015). We then define a series of redshift slices ( $z$-slices) with widths of $\pm 1.5(1+z) \sigma_{|\Delta z| /(1+z)}$. This width is suggested by Malavasi et al. (2016). The $z$-slices are designed in a way that $\gtrsim 90$ per cent of each $z$-slice is overlapping with its next $z$-slice. Such dense design is to appropriately consider the photo- $z$ distribution of galaxies close to the boundaries of each $z$-slice (see section 3.1 of Darvish et al. 2015). The numbers of $z$-slices in different redshift ranges are listed in Table 1 . For each $z$-slice, we calculate the weight for each source, defined as the percentage of the redshift probability distribution function within this $z$-slice. We assign a weight of 100 per cent to sources with available spectroscopic redshifts. To reduce computational time, at each redshift, we only include sources with weight at least 10 per cent. To derive the surface-density field for each $z$-slice, we utilize a two-dimensional (2D) Gaussian kernel whose width adaptively decreases in denser regions, ranging from $\approx 0.2 \mathrm{Mpc}(1$ per cent percentile) to $\approx 0.9 \mathrm{Mpc}$ (99 per cent percentile). The algorithm requires an input 'global smoothing width'. We adopt the value of $0.5 \mathrm{Mpc}$ which is the typical virial radius of X-ray clusters in COSMOS $\left(\log M_{\text {halo }} \approx 13-\right.$ 14, e.g. Finoguenov et al. 2007; George et al. 2011). ${ }^{3}$ Following Darvish et al. (2017), we filter out sources near $(<1 \mathrm{Mpc})$ the edge of the field and/or large masked regions in the COSMOS2015 survey (Laigle et al. 2016), because density measurements for these sources are unreliable. The procedures above yield measurements of surface number density $\left(\Sigma\right.$, in units of $\left.\mathrm{Mpc}^{-2}\right)$ for each source.

We quantify the local environment for each source via the dimensionless overdensity parameter, defined as

$1+\delta=\frac{\Sigma}{\Sigma_{\text {median }}}$,

where $\Sigma_{\text {median }}$ is the median $\Sigma$ at each redshift. To minimize the effects of cosmic variance, $\Sigma_{\text {median }}$ is calculated within $z \pm 0.2$ at redshift $z$. Figs 2 and 3 show the overdensity maps for two $z$-slices

${ }^{3}$ Darvish et al. (2015) tested global smoothing widths from 0.1 to $2.0 \mathrm{Mpc}$ and did not find a significant change in the resulting density field. 

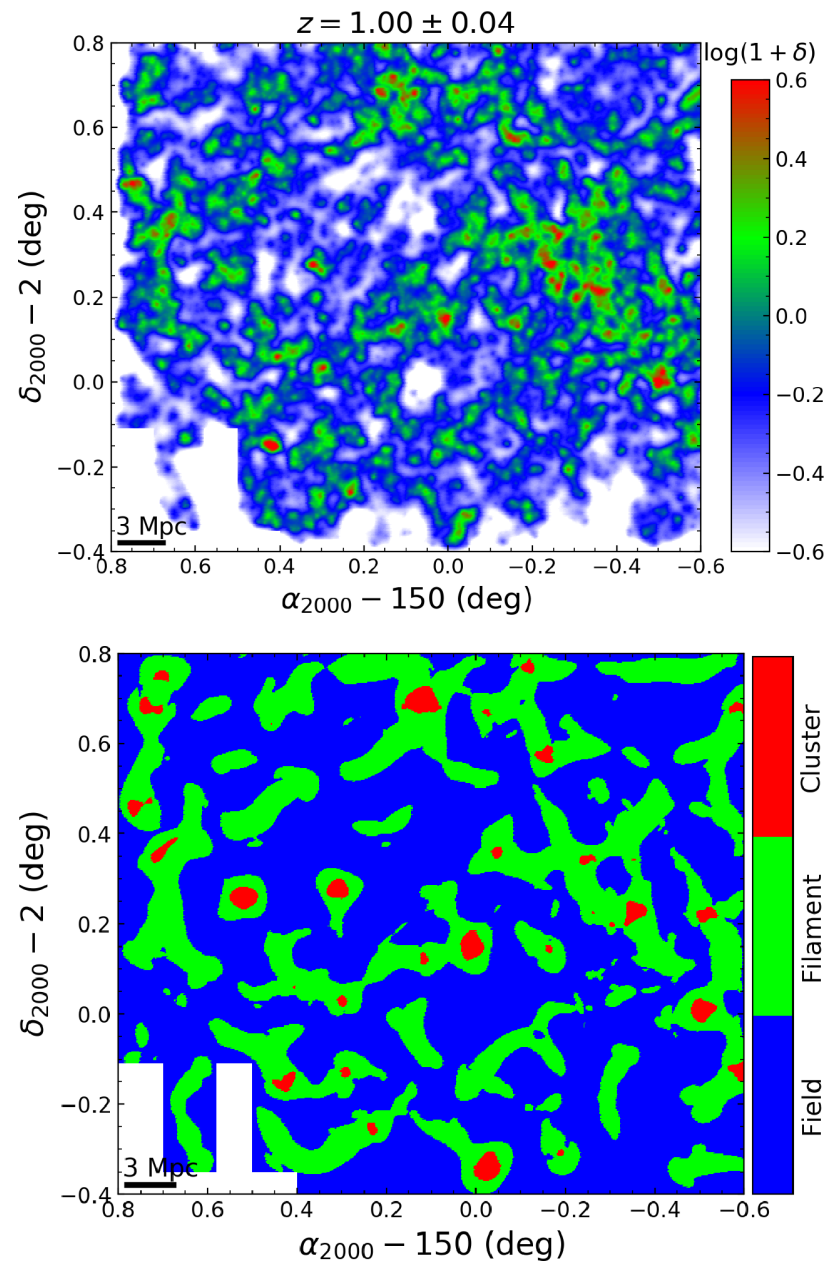

Figure 2. The maps of overdensity (top) and cosmic web (bottom) for the $z$-slice at $z=1.00 \pm 0.04$ derived from our galaxy sample (see Section 2.3). A physical scale of $3 \mathrm{Mpc}$ is marked at the lower left corner in each panel. From the field to cluster environment, the overdensity tends to be higher. However, this is only a statistical trend. For example, high overdensity (top) does not necessarily correspond to cluster (bottom), and vice versa (see Section 2.3.2). The clusters identified in COSMOS are relatively low-mass systems ( $\log M_{\text {halo }} \lesssim 14$; see Section 2.3.1). The white patches at the lower left corner are masked regions where NIR imaging data are not available (McCracken et al. 2012).

centred at $z=1.0$ and 2.0, respectively. The above overdensity measurements are based on sources with $K_{\mathrm{s}}<24$ (see Section 2.1).

Figs 1 and 4 show overdensity as a function of redshift and $M_{\star}$, respectively (see Table 1 for typical overdensity ranges of our sample). There are positive trends between overdensity and $M_{\star}$ at $z \lesssim 2$, consistent with previous work (e.g. Darvish et al. 2017).

In our overdensity estimation above, we have appropriately weighted sources according to their photo- $z$ uncertainties (see Section 2.3). However, this weighting technique does not account for catastrophic photo- $z$ outliers. The outlier fractions are $\approx 0.5$ per cent -10 per cent, when compared with different spec- $z$ catalogues (see Section 2.1 and Table 1). The photo- $z$ outliers increase the noise level in the derivation of density field. Darvish et al. (2015) assessed the effects of outliers via simulations (see their section 5.1). They concluded that an outlier fraction of 10 per cent has little effect on the derived density field. Therefore, our results should not be qualitatively affected by the photo- $z$ outliers.
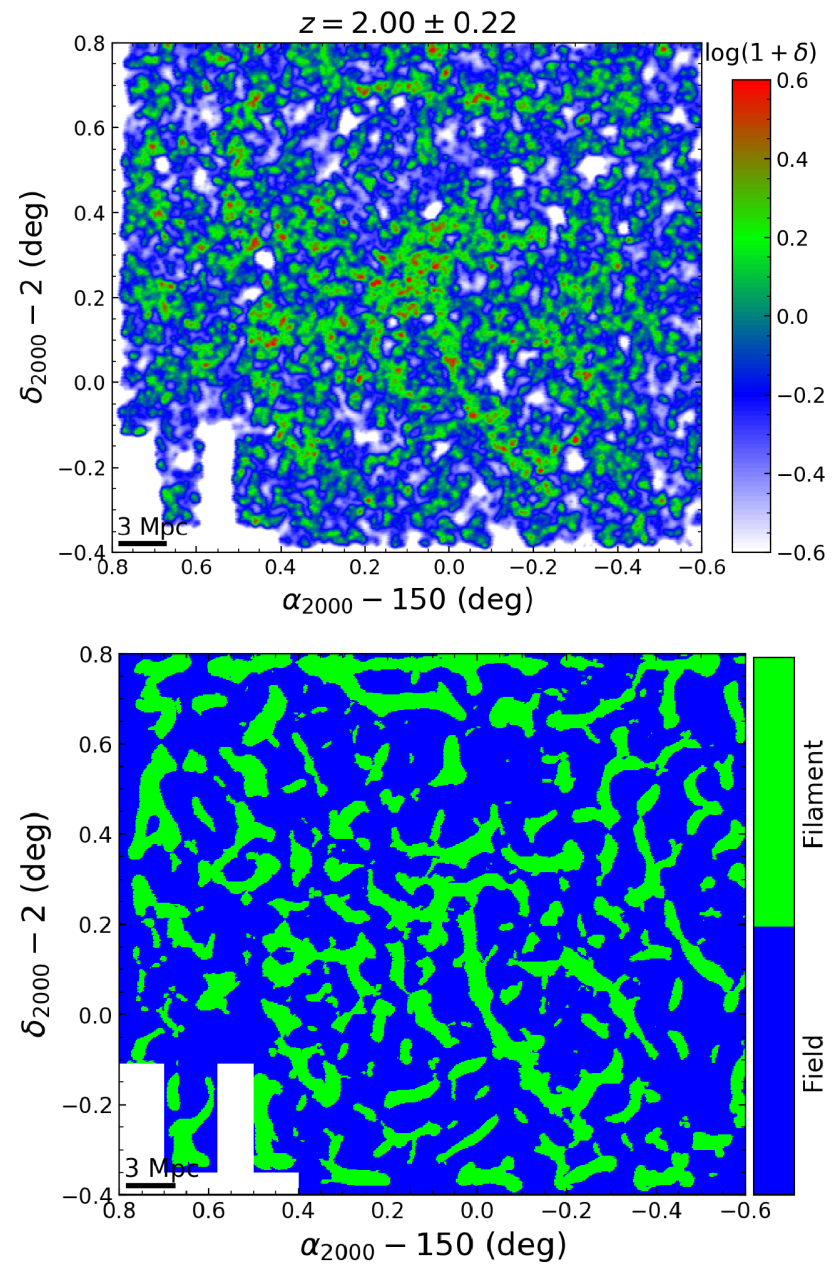

Figure 3. Same format as Fig. 2, but for the $z$-slice centred at $z=2.00 \pm 0.22$. Unlike in Fig. 2, we do not assign cluster environment due to its generally weak signals (see Section 2.3.2).

The typical stellar mass of our sample is relatively small $\left(\log M_{\star} \lesssim 10\right.$; see Table 1$)$. We have also tested using only a subsample of $\log M_{\star}>10$ galaxies when estimating the density field. Our results (Section 3 ) do not change qualitatively. The total stellar mass included in this subsample is $\approx 80$ per cent of that included in the entire sample. However, the subsample consists of only $\approx 30$ per cent of our sources, inevitably leading to stronger Poisson noise in the density-field estimation.

\subsubsection{Cosmic web (global environment)}

Based on the density field derived in Section 2.3.1, we extract a cosmic-web estimate with the multiscale morphology filter (MMF) algorithm (e.g. Aragón-Calvo et al. 2007; Darvish et al. 2014, 2017). The basic idea is to measure the geometry of the density field around each point in the $z$-slice. If the geometry is similar to that of a typical cluster/filament, then the point's environment is classified as cluster/filament; otherwise it is classified as the field.

Specifically, we first derive the Hessian matrix (second-order partial derivatives) of the density field for each point in a $z$-slice (see section 3.4.1 of Darvish et al. 2017 for details). We then calculate two eigenvalues for each Hessian matrix. The eigenvalues describe the density-field geometry around the point. Based on the signs, ratios, and normalizations of the two eigenvalues, a cluster signal 


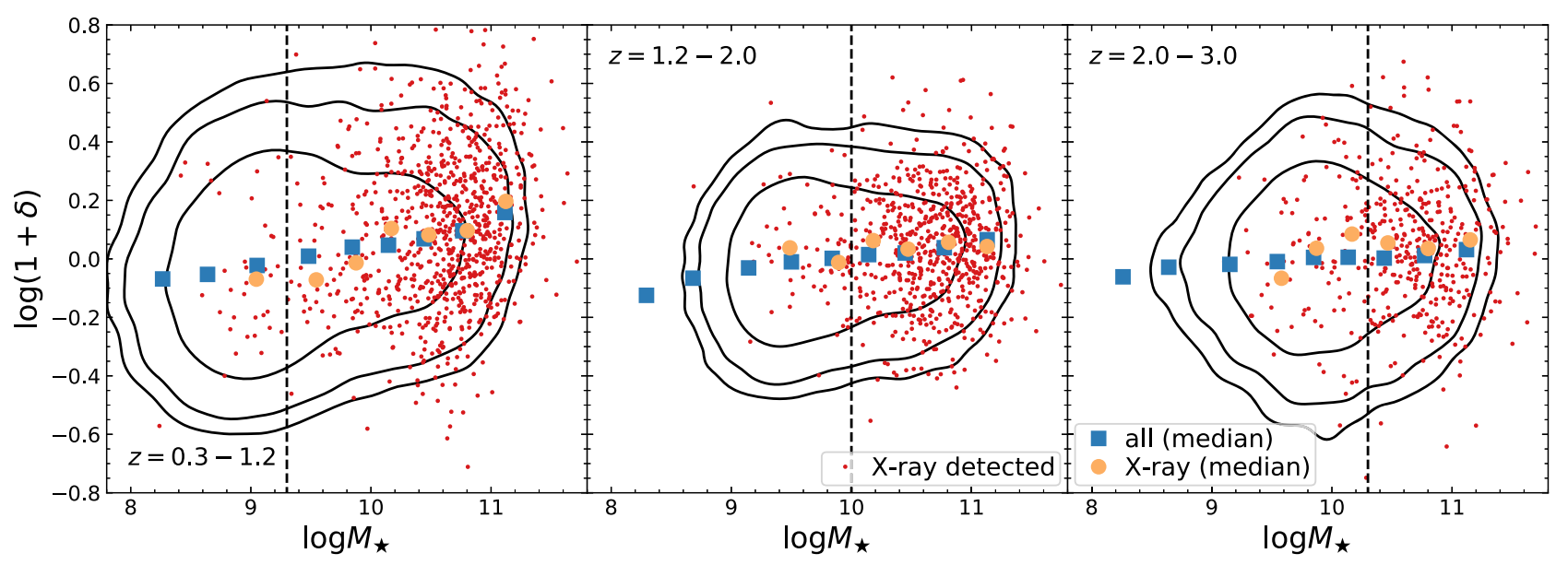

Figure 4. Overdensity versus $M_{\star}$ for different redshift ranges. The contours include 68 per cent, 90 per cent, and 95 per cent of all galaxies, respectively. The red points represent X-ray-detected sources. The blue squares and orange circles indicate the median overdensity in each $M_{\star}$ bin (Section 3.1.1) for all galaxies and X-ray-detected sources, respectively. We only calculate the medians for bins with more than 20 sources to avoid large uncertainties. The vertical dashed lines represent the $M_{\star}$ completeness limits at $z=1.2,2$, and 3, respectively (Section 2.2). The X-ray-detected sources tend to have high $M_{\star}$ but do not show an obvious dependence on overdensity.

$\left(S_{\mathrm{c}}\right)$ and a filament signal $\left(S_{\mathrm{f}}\right)$ are obtained for the point. Here, the signals are two numbers within $0-1$, where larger values indicate higher chances of lying in a cluster/filament. To account for the multiscale nature of clusters and filaments, the above procedures are repeated but each time the density field is smoothed with a Gaussian kernel of different physical scales $(0.25,0.50,0.75,1.00$, 1.50 , and $2.00 \mathrm{Mpc})$. The final cluster/filament signal for each point is assigned as the largest value among those obtained with different smoothing scales.

After obtaining the signal maps for each $z$-slice, we need to apply appropriate signal thresholds to identify clusters and filaments. A higher signal threshold generally increases reliability but decreases completeness in structure selection. We adopt the redshiftdependent thresholds from section 3.4.2 of Darvish et al. (2017), which are designed to balance between reliability and completeness. These thresholds are $T_{\mathrm{c}}=0.0639 z+0.1142$ (cluster) and $T_{\mathrm{f}}=0.0253 z+0.0035$ (filament). Following Darvish et al. (2017), we assign a cluster environment to a point if it has $S_{\mathrm{c}} \geq S_{\mathrm{f}}$ and $S_{\mathrm{c}} \geq T_{\mathrm{c}}$. If a point is not assigned as a cluster environment but has $S_{\mathrm{f}} \geq T_{\mathrm{f}}$, we assign it as a filament environment. If an object is not assigned as cluster or filament environment, we assign it as the field environment.

The above criterion has been successfully applied to $z \lesssim 1.2$ sources (e.g. Darvish et al. 2017). Fig. 2 shows the cosmic-web map for the $z$-slice centred at $z=1.0$. The clusters are roughly round with typical physical sizes of $\sim 1 \mathrm{Mpc}$. The filaments are elongated, with a typical length of $\sim 10 \mathrm{Mpc}$. However, we find, at $z \gtrsim 1.2$, the clusters are often dominated by noise. This is understandable as clusters are still forming at high redshifts and they exist in the form of protoclusters (e.g. Kravtsov \& Borgani 2012; Overzier 2016). The protoclusters have weaker signals and are generally beyond our detection sensitivity. Therefore, we do not assign cluster environments for redshift ranges of $z=1.2-2.0$ and 2.0-3.0. Specifically, if an object at $z=1.2-3.0$ has $S_{\mathrm{f}} \geq T_{\mathrm{f}}$, we assign it as a filament environment; otherwise, we assign it as a field environment. Fig. 3 displays the cosmic-web map for the $z$-slice centred at $z=2.0$. The numbers of sources associated with different cosmicweb environments are summarized in Table 1.

Fig. 5 shows the overdensity as a function of redshift for differ-

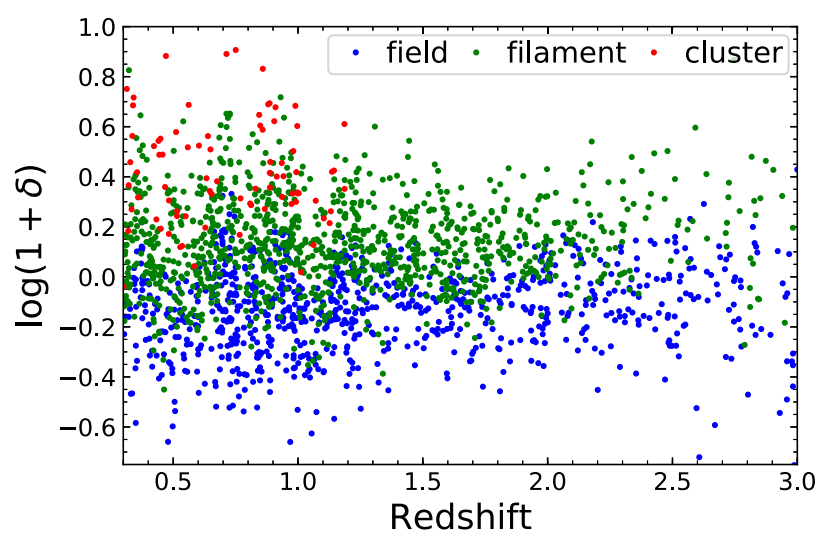

Figure 5. Overdensity versus redshift for 2000 randomly selected sources in our sample. Different colours indicate sources with different cosmicweb environments. The clusters identified in COSMOS are relatively lowmass systems $\left(\log M_{\text {halo }} \lesssim 14\right.$; see Section 2.3.1). We do not assign cluster environment at redshifts above $z=1.2$ due to its generally weak signals (Section 2.3.2). Although the overdensity tends increase moving from the field to clusters, there are significant overlaps between different cosmic-web environments.

ent cosmic-web environments. Although the overdensity generally rises from the field to clusters, there are substantial overlapping areas in the overdensity-redshift parameter space. This overlap is understandable as the overdensity and cosmic-web measurements describe cosmic environment on different scales (sub-Mpc versus $\approx 1-10 \mathrm{Mpc}$ ). The overlap highlights the importance of our MMF algorithm in the construction of the cosmic web, and a simple overdensity-based algorithm would not be feasible for cosmic-web association. Readers might worry that the overlap might smear out potentially weak trends between $\overline{\mathrm{BHAR}}$ and environment. However, this is not an issue in our analyses, because we assess $\overline{\text { BHAR }}$ dependence on both overdensity and cosmic-web environment individually and reach consistent results (see Section 3).

George et al. (2011) have associated galaxies with X-ray-selected clusters at $z<1$ using a probabilistic method. We match their cluster-member candidates (member probability above 70 per cent) 
to our sample with a 0.5 arcsec matching radius. As expected, the 1851 matched galaxies generally have high overdensity values of $\log (1+\delta)=0.34-0.66(25-75$ per cent percentile $)$ compared to our overall sample (see Table 1). For these 1851 galaxies, 44 per cent and 50 per cent are assigned as cluster and filament, respectively, in our catalogue. The 44 per cent filament objects tend to lie in the boundary between clusters and filaments in our $z$-slices, where the cluster/filament classification is sensitive to the methodology. The other 6 per cent of galaxies are assigned as the field environment in our catalogue. This disagreement is likely caused by the differences in adopted redshift measurements, as these galaxies' redshift values in the two catalogues differ by $\approx 3$ per cent. In comparison, the other 94 per cent of galaxies (George et al. 2011 cluster candidates assigned as cluster/filament galaxies) have redshift differences of only $\approx 0.7$ per cent. Our adopted photo- $z$ values should have improved quality compared to those adopted in George et al. (2011), who used photo- $z$ from an earlier COSMOS catalogue (Ilbert et al. 2009). Note that it is natural that most of our cluster galaxies are not identified by George et al. (2011), because their X-ray-selected clusters are not complete.

\subsection{Black hole accretion rate}

We derive $\overline{\mathrm{BHAR}}$ for samples of sources broadly following the procedures in section 2.3 of Yang et al. (2017). Briefly, we first calculate the total sample-averaged $L_{\mathrm{X}}\left(\overline{L_{\mathrm{X}}}\right)$ considering both X-ray-detected sources (Section 2.4.1) and undetected sources (Section 2.4.2). The undetected sources are considered with an X-ray stacking technique. The stacking procedure is necessary to avoid biases due to the limited X-ray survey sensitivity, as faint AGNs become undetected toward high redshift. We obtain the AGN $\overline{L_{X}}$ by subtracting the $\overline{L_{X}}$ component contributed by XRBs (Section 2.4.3). Finally, we convert AGN $\overline{L_{\mathrm{X}}}$ to $\overline{\mathrm{BHAR}}$ in units of $\mathrm{M}_{\odot} \mathrm{yr}^{-1}$.

\subsubsection{X-ray-detected sources}

We select all X-ray-detected sources using the COSMOS-Legacy X-ray survey (Civano et al. 2016). The COSMOS-Legacy survey, conducted by Chandra, is the deepest X-ray survey available for COSMOS, and can sample most of cosmic accretion power. For instance, at $z \approx 1.5-2.5$, the peak of cosmic AGN activity, it covers $L_{\mathrm{X}}$ ranging from $\approx 10$ times below the knee luminosity of the $\mathrm{X}$ ray luminosity function to $\approx 3$ times above the knee luminosity, corresponding to $\approx 80$ per cent of the total $L_{\mathrm{X}}$ (integrated from the $\mathrm{X}$-ray luminosity function).

There are $\approx 2020$ X-ray sources matched to the optical/NIR COSMOS2015 catalogue based on a likelihood-ratio technique by Marchesi et al. (2016a, see Table 1). Most ( $\gtrsim 90$ per cent) of these $\mathrm{X}$-ray sources should be AGNs considering their relatively high Xray luminosity $\left(\log L_{X}>42.5\right.$, e.g. Xue et al. 2016; Luo et al. 2017). Besides the $\approx 240 \mathrm{BL}$ AGNs, there are $\approx 730 \mathrm{X}$-ray sources with spec- $z$ measurements (see Section 2.1). We use these $\approx 730$ nonBL X-ray sources to assess the photo- $z$ quality for the $\approx 1050 \mathrm{X}$-ray sources with only photo- $z$ measurements, because Yang et al. (2018) estimated most ( $\approx 80$ per cent) of the photo- $z$ sources should be nonBL AGNs. The photo- $z$ have $\sigma_{\mathrm{NMAD}}=0.018$ and $\eta=7.5$ per cent (see Section 2.1), comparable to the AGN photo- $z$ quality in the literature (e.g. Luo et al. 2010; Hsu et al. 2014; Yang et al. 2014).

A source might be detected in multiple X-ray bands. In this case, we choose, in order of priority, hard-band $(2-7 \mathrm{keV})$, fullband $(0.5-7 \mathrm{keV})$, and soft-band $(0.5-2 \mathrm{keV})$ fluxes, for the $L_{\mathrm{X}}$
Table 3. $p$-values (significances) of PCOR analyses (see Section 3.1.2).

\begin{tabular}{|c|c|c|c|}
\hline \multicolumn{4}{|c|}{$z=0.3-1.2(48581$ galaxies $)$} \\
\hline Relation & Pearson & Spearman & Kendall \\
\hline$\overline{\mathrm{BHAR}}$-overdensity & $0.9(0.1 \sigma)$ & $0.2(1.2 \sigma)$ & $0.6(0.6 \sigma)$ \\
\hline$\overline{\mathrm{BHAR}}-M_{\star}$ & $10^{-48}(14.7 \sigma)$ & $10^{-45}(14.1 \sigma)$ & $10^{-10}(6.3 \sigma)$ \\
\hline \multicolumn{4}{|c|}{$z=1.2-2.0(18944$ galaxies $)$} \\
\hline Relation & Pearson & Spearman & Kendall \\
\hline$\overline{\mathrm{BHAR}}$-overdensity & $0.08(1.7 \sigma)$ & $0.4(0.8 \sigma)$ & $0.4(0.8 \sigma)$ \\
\hline$\overline{\mathrm{BHAR}}-M_{\star}$ & $10^{-33}(12.0 \sigma)$ & $10^{-21}(9.5 \sigma)$ & $10^{-7}(5.0 \sigma)$ \\
\hline \multicolumn{4}{|c|}{$z=2.0-3.0$ (5932 galaxies) } \\
\hline Relation & Pearson & Spearman & Kendall \\
\hline$\overline{\mathrm{BHAR}}-$ overdensity & $0.08(1.8 \sigma)$ & $0.1(1.6 \sigma)$ & $0.4(0.9 \sigma)$ \\
\hline$\overline{\mathrm{BHAR}}-M_{\star}$ & $10^{-19}(8.9 \sigma)$ & $10^{-19}(9.0 \sigma)$ & $10^{-5}(4.3 \sigma)$ \\
\hline
\end{tabular}

Note. Here, the numbers of galaxies are different from Table 1, because only sources above the limiting $M_{\star}$ are used in the analyses of the $\overline{\mathrm{BHAR}}-M_{\star}$ environment relation (see Section 3.1.1).

calculation below. This order of detection bands is to minimize the effects of X-ray obscuration. The fractions of X-ray sources with fluxes from the hard, full, and soft bands are 63 per cent, 34 per cent, and 3 per cent, respectively.

The X-ray fluxes in the COSMOS-Legacy catalogue are only corrected for Galactic absorption. Marchesi et al. (2016a) have estimated intrinsic absorption column densities $\left(N_{\mathrm{H}}\right)$ based on hardness ratios. We do not apply these absorption corrections, because the majority ( $\approx 70$ per cent) of sources have poorly constrained $N_{\mathrm{H}}$ values (consistent with zero at a 90 per cent confidence level) mainly due to limited numbers of counts. Instead, we evaluate the level of absorption corrections for COSMOS-like sources using the ultradeep 7 Ms catalogue of the Chandra Deep Field-South (CDF-S; Luo et al. 2017). Such sources have $\approx 44$ times more counts in CDF-S than COSMOS and absorption corrections have been estimated individually (e.g. Yang et al. 2016; Liu et al. 2017; Luo et al. 2017). These COSMOS-like sources are selected via applying the COSMOS flux limits (Civano et al. 2016) to CDF-S. We find that these COSMOS-like sources have a median absorption correction factor (intrinsic flux divided by observed flux) of $\approx 1.2$. The corresponding uncertainty caused by absorption correction is generally smaller than the statistical uncertainties of $\overline{\mathrm{BHAR}}$ (Section 2.4.3), and thus absorption should not bias our conclusions. The relatively low level of absorption corrections is mainly due to our choice of bands. Another reason is that we can sample ultra-hard $(\approx 10-20 \mathrm{keV}$, rest frame) penetrating X-rays for most sources (see Table 1).

We convert the X-ray fluxes to $L_{\mathrm{X}}$ assuming a power-law model with a photon index of $\Gamma=1.7$, which is the typical intrinsic slope of distant AGNs (e.g. Marchesi et al. 2016b; Yang et al. 2016; Liu et al. 2017). Our conclusions do not change if we adopt a slightly different $\Gamma$ value (e.g. $\Gamma=1.4$ ). The resulting $L_{X}$ values as a function of redshift are displayed in Fig. 1 (also see Table 1 for typical $L_{X}$ ranges). Fig. 1 also shows the estimated $L_{X}$ limit, assuming a $0.5-$ $10 \mathrm{keV}$ flux threshold of $8.9 \times 10^{-16} \mathrm{erg} \mathrm{cm}^{-2} \mathrm{~s}^{-1}$ (Civano et al. 2016).

\subsubsection{X-ray-undetected sources}

We perform X-ray stacking to calculate $\overline{L_{X}}$ for X-ray-undetected sources in our samples. We use the full-band X-ray data. The full band is the most sensitive in the sense that it detects the largest number of X-ray sources (Civano et al. 2016), while the hard band 

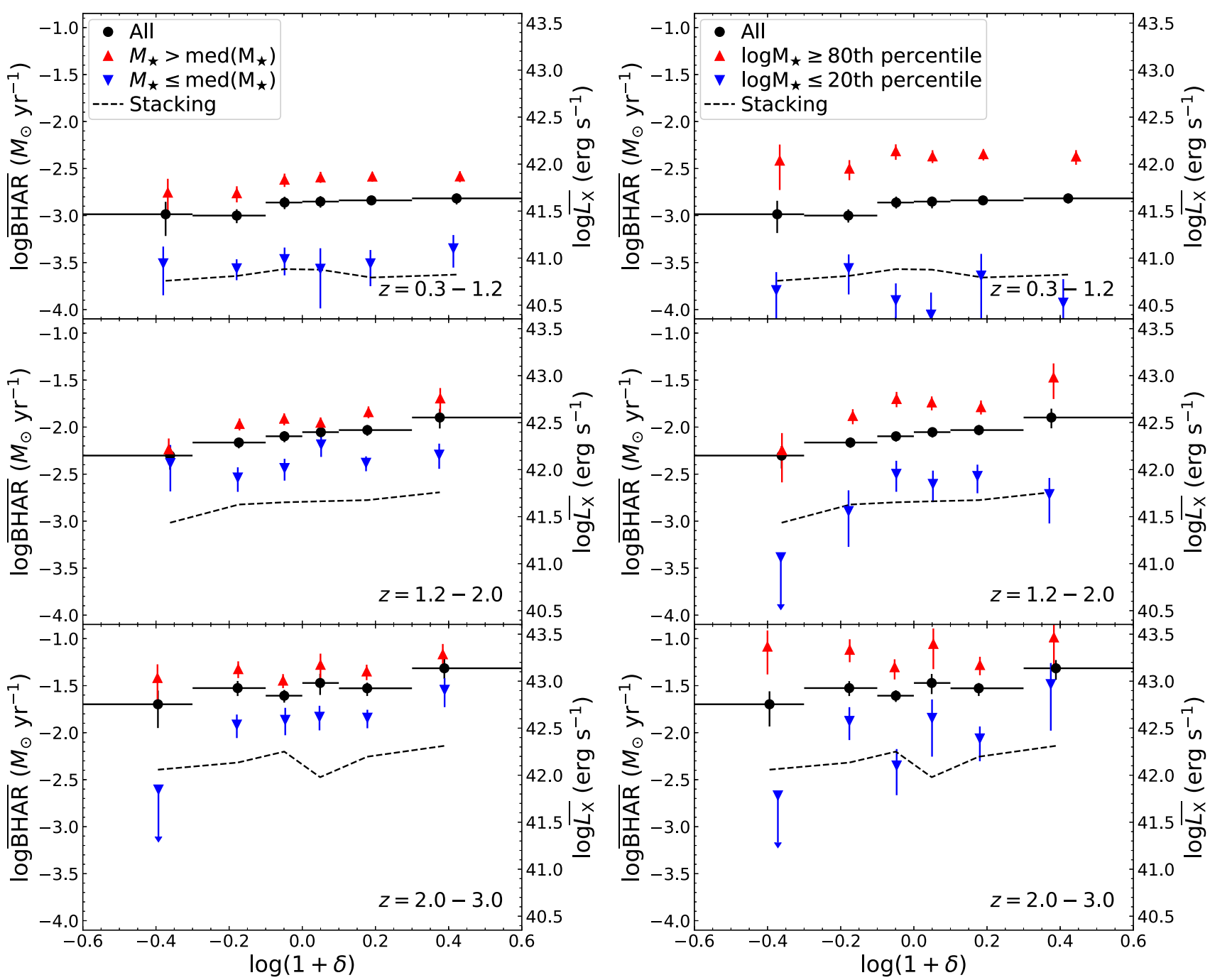

Figure 6. $\overline{\mathrm{BHAR}}$ as a function of overdensity for different redshift ranges. In the left-hand panels, each overdensity bin is split into two subsamples with $M_{\star}$ above and below the median value, respectively. In the right-hand panels, the subsamples include sources with the highest 20 per cent of $M_{\star}$ and the lowest 20 per cent of $M_{\star}$, respectively. $\overline{L_{X}}$ is marked on the right-hand side of each panel. The red upward and blue downward triangles indicate the subsamples with $M_{\star}$ above and below the median value, respectively. The error bars are derived from a bootstrapping technique (see Section 2.4.3). The dashed curves indicate $\overline{\mathrm{BHAR}}$ contributed from X-ray stacking (see Section 2.4.2). The high- $M_{\star}$ subsamples have significantly higher $\overline{\mathrm{BHAR}}$ than the corresponding low- $M_{\star}$ subsamples.

is the least sensitive. Also, compared to the soft band, the full band is less affected by X-ray obscuration. Table 1 shows the rest-frame energy ranges corresponding to the full band. Using the soft or hard band for stacking does not change our conclusions qualitatively.

Based on the full-band X-ray image and exposure map, we broadly follow the procedures in section 2 of Vito et al. (2016). First, we mask the X-ray image for both detected extended and point-like sources. Since the extended-source catalogue for the COSMOSLegacy survey is not available, we use the X-ray cluster catalogue from XMM-Newton observations of COSMOS (Finoguenov et al. 2007). For point-like sources, we use the catalogue from Civano et al. (2016). Since the X-ray clusters are masked, we cannot perform stacking analyses for some of the densest environments, and this could potentially bias our results for $\overline{\mathrm{BHAR}}$ dependence on environment. However, most of the $\overline{\mathrm{BHAR}}$ in our sample is contributed by the X-ray-detected sources (see Section 3.1.1). Indeed, our qualitative results do not change even if we only consider $\overline{\mathrm{BHAR}}$ contributed from X-ray detected sources. Therefore, we argue that the masking of X-ray clusters, and other technical details of the stacking, should not be critical to our analyses (see Section 3).

For each detected source (including extended and point-like sources), we mask its surrounding area with a radius of $R_{\mathrm{msk}}$. We adopt $R_{\mathrm{msk}}=r_{500}$ for extended sources, where $r_{500}$, in the range of $\approx 0.5-3 \mathrm{arcmin}$, is the estimated cluster radius provided by Finoguenov et al. (2007). We adopt $R_{\mathrm{msk}}=20$ arcsec for point-like sources, as Vito et al. (2016) show such a radius is large enough to include nearly all X-ray flux even for the brightest sources (thousands of counts) at the largest off-axis angles. We do not adopt a masking radius that depends on off-axis angle, because one source is often observed by multiple pointings and has different off-axis angles in different pointings. The masked regions (including those for extended and point-like sources) cover a total of $\approx 20$ per cent of the survey area. We fill each masked region with the background randomly sampled from the corresponding background region, defined as the annulus with inner and outer radii of $R_{\mathrm{msk}}$ and $2 R_{\mathrm{msk}}$, respectively. This is performed with the 'dmfilth' command in the 

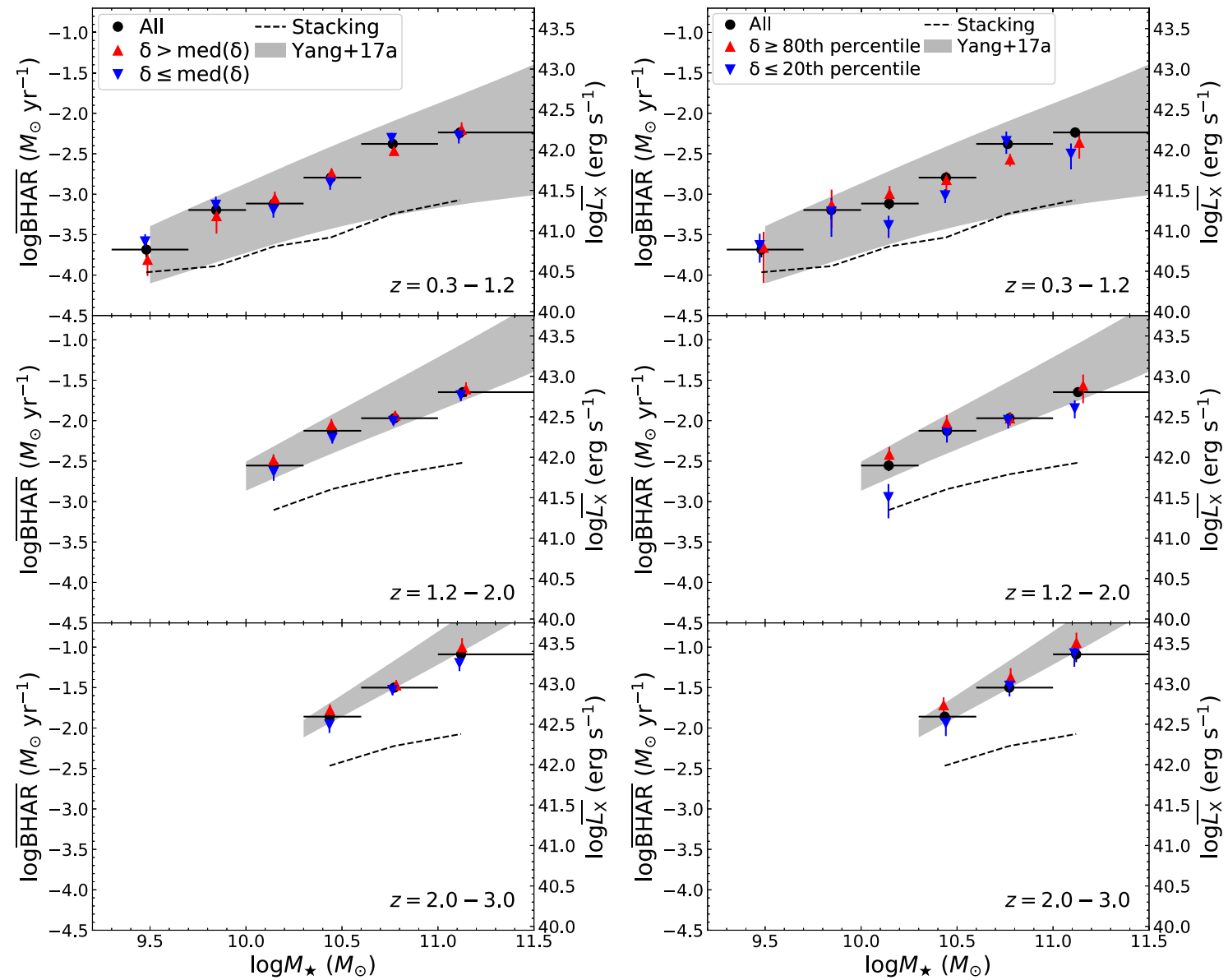

Figure 7. Same format as Fig. 6 but for $\overline{\mathrm{BHAR}}$ versus $M_{\star}$. In both panels, the shaded regions show the $\overline{\mathrm{BHAR}}-M_{\star}$ relations from Yang et al. (2018). The lower and upper boundaries of the Yang et al. (2018) relations correspond to the $\overline{\mathrm{BHAR}}$ at the low and high limits of the redshift bin, respectively, except for the redshift bin of $z=0.3-1.2$. For $z=0.3-1.2$, the lower boundary of the shaded region represents $\overline{\text { BHAR }}$ at $z=0.4$, which is the lowest redshift probed in Yang et al. (2018). The $\overline{\mathrm{BHAR}}$ for high- and low-overdensity subsamples are similar in general.

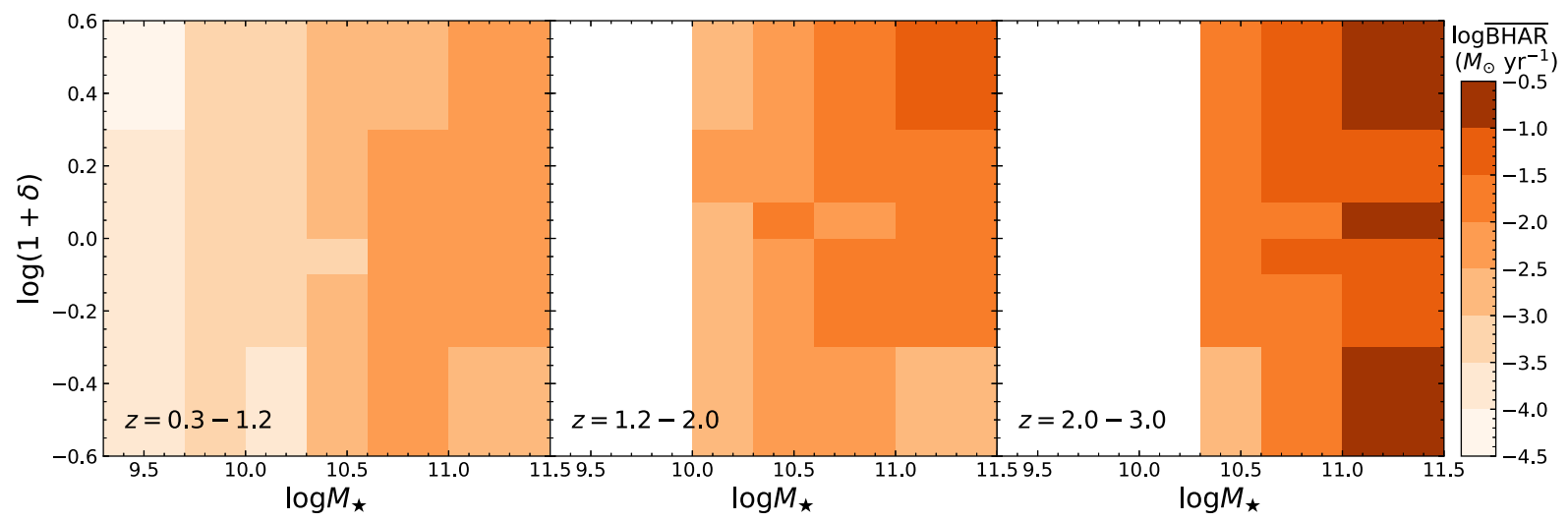

Figure 8. $\overline{\mathrm{BHAR}}$ as a function of overdensity and $M_{\star}$ for different redshift ranges. Darker colour indicates higher $\overline{\mathrm{BHAR}}$ as labelled. White colour indicates $\overline{\mathrm{BHAR}}$ is not available, because of large uncertainties on $\overline{\mathrm{BHAR}}$ or $M_{\star}$ lying below the completeness limits. The $\overline{\mathrm{BHAR}}$ in each bin has an uncertainty of $\lesssim 0.3$ dex.

Chandra data-analysis package CIAO. In the analyses below, we treat the masked regions as background.

We then derive net count rates for X-ray-undetected galaxies (Section 2.1) utilizing the masked X-ray image and exposure map. We only calculate the net count rates for each source that is not within or close to the masked regions, i.e. its distance $(d)$ to every masked source should satisfy $d>R_{\text {msk }}+R_{\text {phot }}$, where $R_{\text {phot }}$ is the radius used to perform $\mathrm{X}$-ray photometry. About 30 per cent of sources are discarded in this step, and we account for X-ray emission from these sources in Section 2.4.3.

For each object, we obtain its total X-ray counts enclosed within a radius of $R_{\text {phot }}=4$ arcsec and the average exposure time for this 

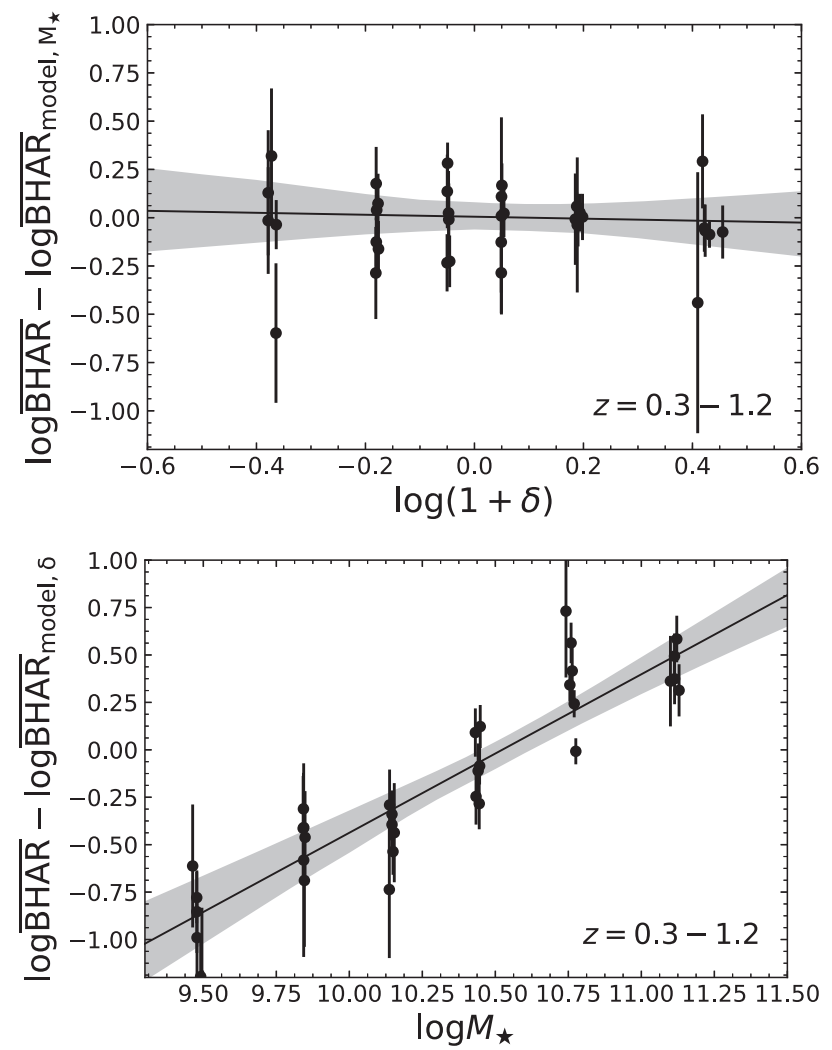

Figure 9. $\overline{\mathrm{BHAR}}$ residuals (of the $\overline{\mathrm{BHAR}}-M_{\star}$ fit) versus overdensity (top) and $\overline{\mathrm{BHAR}}$ residuals (of the $\overline{\mathrm{BHAR}}$-overdensity fit) versus $M_{\star}$ (bottom) for $z=0.3-1.2$. The black lines represent the best fit of the data points; the shaded regions indicate the $3 \sigma$ uncertainties. In the top panel, the residual $\overline{\text { BHAR }}$ is relatively flat as a function of overdensity; in the bottom panel, the residual $\overline{\mathrm{BHAR}}$ rises steeply toward high $M_{\star}$. Results are found similar at $z=1.2-2.0$ and 2.0-3.0.

area. The value $R_{\text {phot }}=4$ arcsec is chosen because the signal-tonoise ratio of stacking becomes substantially lower for larger $R_{\text {phot }}$ values. We calculate the total count rate by dividing the total counts by the average exposure time in the $R_{\text {phot }}$ circle. The total count rate includes not only the $\mathrm{X}$-ray emission from the source but also the background. Therefore, we need to subtract the background properly. We choose the background region as an annulus with inner and outer radii of 10 and 20 arcsec, respectively, and calculate the background count rate. We obtain the net count rates by subtracting the background count rates from the total count rates.

Since we are performing aperture photometry with a limited aperture size $\left(R_{\text {phot }}=4 \operatorname{arcsec}\right)$, there is a fraction of X-ray emission falling outside of our photometric aperture. To estimate this effect, we recalculate net counts but with a very large photometric radius of $R_{\text {phot }}=10 \operatorname{arcsec}$. We find that the final stacked count rates for $R_{\text {phot }}=10$ arcsec are systematically higher than those for $R_{\text {phot }}=4$ arcsec by a factor of $\approx 1.4$. Thus, $R_{\text {phot }}=4 \operatorname{arcsec}$ corresponds to a radius for $1 / 1.4 \approx 70$ per cent encircled-energy fraction (EEF) on average. The 70 per cent EEF is reasonable considering that the typical 50 per cent EEF radius is 3-4 arcsec for the detected sources (see fig. 2 of Civano et al. 2016). We correct the net count rate $\left(R_{\text {phot }}=4 \operatorname{arcsec}\right)$ for each source by multiplying by 1.4 to obtain the final net count rate.

Following Yang et al. (2017), we obtain the average count rate for samples of sources and convert it to full-band X-ray flux with a constant factor $\left(9.5 \times 10^{-12} \mathrm{erg} \mathrm{cm}^{-2}\right.$ counts $\left.^{-1}\right)$. The factor is calculated with PIMMS assuming $\Gamma=1.7$ (Section 2.4.1). We derive the average $\mathrm{X}$-ray luminosity $\left(\overline{L_{\mathrm{X}, \text { stack }}}\right)$ from the average flux and the average redshift of the stacked sample. Our conclusions do not change if we adopt $\Gamma=1.4$ for X-ray-undetected sources (resulting in a $\approx 10$ per cent change of $\overline{L_{\mathrm{X} \text {, stack }}}$ at $z=1$ ), as expected from the fact that total X-ray emission is dominated by X-ray-detected sources in general.

\subsubsection{Calculation of $\overline{\mathrm{BHAR}}$}

We calculate $\overline{\mathrm{BHAR}}$ for samples of sources following the recipe in section 2.3 of Yang et al. (2017). We first calculate the average AGN X-ray luminosity for the sample as

$\overline{L_{\mathrm{X}}}=\frac{\left(\Sigma_{\mathrm{det}} L_{\mathrm{X}}\right)+N_{\text {non }} \overline{L_{\mathrm{X}, \mathrm{stack}}}-\Sigma_{\mathrm{all}} L_{\mathrm{X}, \mathrm{XRB}}}{N_{\mathrm{det}}+N_{\mathrm{non}}}$,

where $N_{\text {det }}$ and $N_{\text {non }}$ are numbers of X-ray-detected and undetected sources, respectively; $L_{\mathrm{X}}$, XRB is the luminosity from XRBs.

In the numerator of equation (2), the first term $\left(\Sigma_{\mathrm{det}} L_{\mathrm{X}}\right)$ is the total luminosity of X-ray-detected sources (Section 2.4.1). The second term $\left(N_{\text {non }} \overline{L_{\mathrm{X} \text {,stack }}}\right)$ accounts for the total luminosity of X-rayundetected sources. Stacked sources are only a subsample of the undetected sources as some undetected sources (within or close to the masked regions) are discarded in the stacking procedure (see Section 2.4.2). The formula $\left(N_{\text {non }} \overline{L_{\mathrm{X} \text {,stack }}}\right)$ assumes these discarded sources have the same average luminosity as the stacked sources.

The third term $\left(\Sigma_{\text {all }} L_{\mathrm{X}, \mathrm{XRB}}\right.$ in equation 2$)$ is to subtract the XRB component from the total luminosity. For star-forming galaxies (see Section 2.2 for the classification), we use $L_{\mathrm{X}, \mathrm{XRB}}=\alpha M_{\star}+\beta \mathrm{SFR}$. The coefficients ( $\alpha$ and $\beta$ ) are functions of redshift from model 269 of Fragos et al. (2013); and model 269 is a theoretical XRB model which is preferred by observations of galaxies at $z \approx 0-2$ (Lehmer et al. 2016; typical uncertainties $\lesssim 0.3 \mathrm{dex}$ ). The $M_{\star}$ value is from our SED fitting (Section 2.2). We approximate SFR by using the value from the star-forming main sequence in equation (6) of Aird, Coil \& Georgakakis (2017). For quiescent galaxies, we neglect the $\mathrm{SFR}$ term and estimate their XRB emission as $L_{\mathrm{X}}, \mathrm{XRB}=\alpha M_{\star}$, since this term dominates. The sample-averaged $\mathrm{XRB}$ emission is $\approx 0.6-$ 1.3 dex lower than the average AGN emission. It is even $\approx 0.3 \mathrm{dex}$ lower than the stacked X-ray emission. Therefore, the details of subtracting the XRB component are not critical to our analyses.

Following Yang et al. (2017), we convert $\overline{L_{X}}$ to BHAR as

$\overline{\mathrm{BHAR}}=\frac{3.53 \overline{L_{\mathrm{X}}}}{10^{45} \mathrm{erg} \mathrm{s}^{-1}} \mathrm{M}_{\odot} \mathrm{yr}^{-1}$.

This conversion assumes a constant bolometric correction factor $\left(k_{\mathrm{bol}}=22.4\right.$; Vasudevan \& Fabian 2007) and a constant radiation efficiency $(\epsilon=0.1)$. We calculate the uncertainties of $\overline{\text { BHAR }}$ with a bootstrapping technique (see section 2.3 of Yang et al. 2017). The bootstrapping $\overline{\mathrm{BHAR}}$ errors are statistical uncertainties resulting from finite sampling.

A radiation efficiency of $\epsilon=0.1$ is a typical value for the overall AGN population and is supported by observations (e.g. Marconi et al. 2004; Davis \& Laor 2011; Brandt \& Alexander 2015). Studies have found $k_{\mathrm{bol}}$ depends on AGN luminosity (e.g. Steffen et al. 2006; Hopkins, Richards \& Hernquist 2007; Lusso et al. 2012). We do not adopt a $L_{\mathrm{X}}$-dependent $k_{\mathrm{bol}}$, because it cannot be applied to our stacking procedure. Also, a $L_{\mathrm{X}}$-dependent $k_{\text {bol }}$ requires careful subtraction of non-negligible XRB contributions for individual lowluminosity AGNs, and this task is challenging and beyond our work. 
Table 4. AGN fractions (per cent) in different overdensity bins.

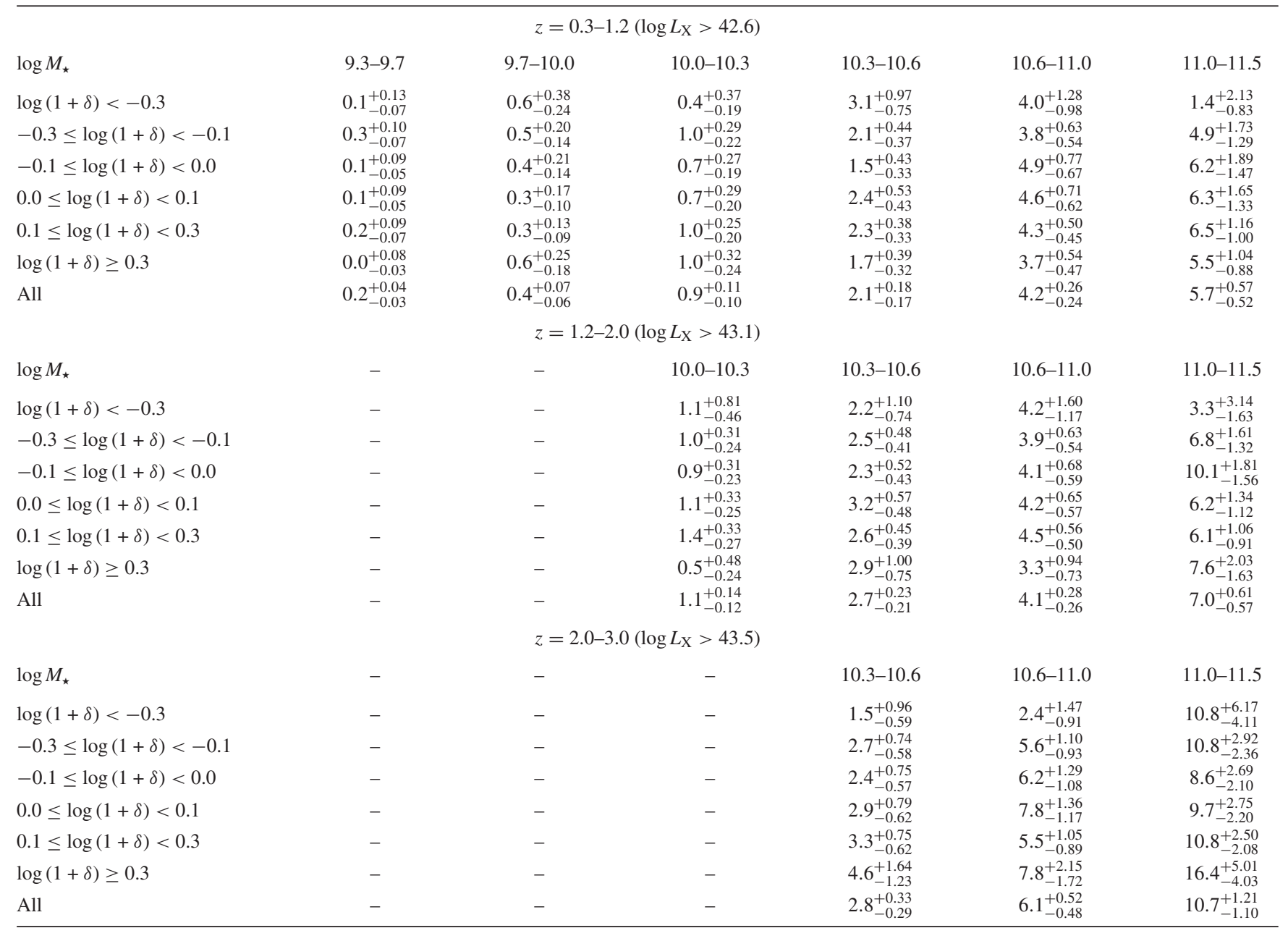

Therefore, we adopt the constant $k_{\mathrm{bol}}$ for simplicity and consistency. However, we have tested applying a $L_{\mathrm{X}}$-dependent $k_{\mathrm{bol}}$ (Hopkins et al. 2007) for AGN-dominated $X$-ray sources with $\log L_{X}>43$ and our results do not change qualitatively. This is as expected, because our main conclusions only depend on the relative values of $\overline{\mathrm{BHAR}}$ in different environments which are not significantly affected by different $k_{\text {bol }}$ schemes. Admittedly, there might be systematics up to a factor of a few in our absolute values of $\overline{\mathrm{BHAR}}$ due to the uncertainties of $k_{\mathrm{bol}}$ and $\epsilon$. We have also marked $\overline{L_{\mathrm{X}}}$ values in the relevant figures below, allowing readers to consider either $\overline{\mathrm{BHAR}}$ or $\overline{L_{X}}$ when viewing these.

\section{RESULTS}

\section{1 $\overline{\text { BHAR }}$ versus overdensity}

\subsubsection{Qualitative tests}

To probe the $\overline{\mathrm{BHAR}}$ dependence on overdensity at different redshifts, we first bin sources into redshift ranges of $0.3-1.2,1.2-2.0$, and 2.0-3.0, respectively. We restrict our analyses to sources above the $M_{\star}$ completeness limits, $\log M_{\star}=9.3,10$, and 10.3, for redshift ranges of $0.3-1.2,1.2-2.0$, and 2.0-3.0, respectively (see Figs 1 and 4). Applying these $M_{\star}$ cuts is crucial, because incomplete $M_{\star}$ samples could lead to biased $\overline{\mathrm{BHAR}}$ values. For example, the $\overline{\mathrm{BHAR}}$ at $\log M_{\star} \approx 8$ in the bin of $z=0.3-1.2$ would be strongly biased to $z \lesssim 0.5$, above which $\log M_{\star} \approx 8$ galaxies remain largely undetected (see Fig. 1, top). Therefore, such a $\overline{\mathrm{BHAR}}$ value would not be representative of the entire redshift range of $z=0.3-1.2$. Table 3 lists the sizes of these refined samples in different redshift ranges. Hereafter, this rule applies to all the analyses, unless otherwise stated. The fractions of our X-ray detected sources lying above the $M_{\star}$ cuts are 96 per cent, 90 per cent, and 77 per cent for redshift ranges of $0.3-1.2,1.2-2.0$, and 2.0-3.0, respectively (see Fig. 4). Therefore, we are still capturing most accretion power after applying the $M_{\star}$ cuts. For each redshift bin, we further divide the sources into overdensity bins of $\log (1+\delta)<-0.3, \log (1+\delta)=-(0.3-0.1), \log (1$ $+\delta)=-0.1-0, \log (1+\delta)=0-0.1, \log (1+\delta)=0.1-0.3$, and $\log (1+\delta)>0.3$. These bin boundaries $(-0.3,-0.1,0,0.1$, and 0.3 ) roughly correspond to $\approx 10$ per cent, 30 per cent, 50 per cent, 70 per cent, and 90 per cent percentiles of the $\log (1+\delta)$ distribution of all objects.

We calculate $\overline{\mathrm{BHAR}}$ with the methods in Section 2.4.3 for all the bins and show the results in Fig. 6. $\overline{\mathrm{BHAR}}$ tends to be slightly higher toward high overdensity (black points), likely due to the positive dependence between $M_{\star}$ and overdensity and the intrinsic BHAR$M_{\star}$ correlation (see Fig. 4; e.g. Xue et al. 2010; Georgakakis et al. 2017; Yang et al. 2017, 2018; Aird, Coil \& Georgakakis 2018). To show the $\overline{\mathrm{BHAR}}$ dependence on $M_{\star}$, we divide each overdensity sample into high- $M_{\star}$ and low- $M_{\star}$ subsamples. In Fig. 6 (left), 


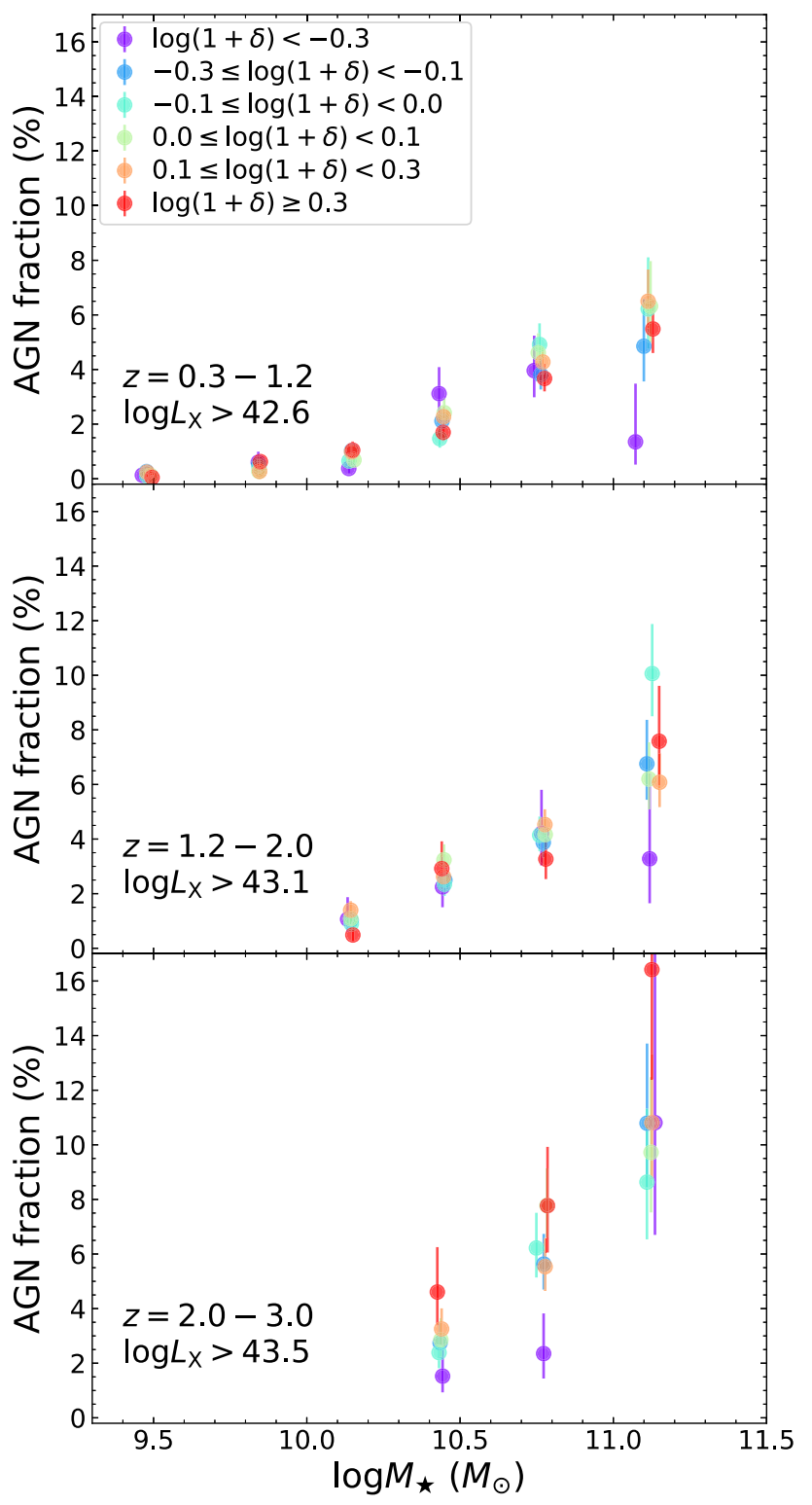

Figure 10. AGN fraction as a function $M_{\star}$ for overdensity bins. The data are from Table 4 . At a given $M_{\star}$, different overdensity bins have similar AGN fractions. Some of the data points are overlapping due to this similarity.

the high- $M_{\star}$ and low- $M_{\star}$ subsamples have $M_{\star}$ above and below the median $M_{\star}$ of the overdensity sample, respectively; in Fig. 6 (right), the high- $M_{\star}$ and low- $M_{\star}$ subsamples include sources with the highest 20 per cent $M_{\star}$ and the lowest 20 per cent $M_{\star}$ of the overdensity sample, respectively. In both the left- and right-hand panels, the high- $M_{\star}$ subsamples have significantly higher $\overline{\mathrm{BHAR}}$ than their corresponding low- $M_{\star}$ subsamples, indicating the previously known strong $\overline{\mathrm{BHAR}}-M_{\star}$ correlation. The $\overline{\mathrm{BHAR}}$ differences between the high- $M_{\star}$ and low- $M_{\star}$ subsamples are generally larger in the right-hand panels than in the corresponding left-hand panels, as expected from the positive $\overline{\mathrm{BHAR}}-M_{\star}$ correlation.

To investigate the potential $\overline{\mathrm{BHAR}}-$ overdensity correlation for the $M_{\star}$ controlled sample, we divide the sources into bins of $\log M_{\star}=9.3-9.7,9.7-10,10-10.3,10.3-10.6,10.6-11$, and 1111.5 for each redshift range. The bin widths are $\approx 0.4$ dex and the $M_{\star}$ limits at $z=1.2,2.0$, and 3.0 (Section 2.2) are chosen as the boundaries of the $M_{\star}$ bins. We then split each $M_{\star}$ sample into highand low-overdensity subsamples in a similar way as in Fig. 6.

We calculate $\overline{\mathrm{BHAR}}$ for all the $M_{\star}$ samples and overdensity subsamples. The results are shown in Fig. 7 (left). The high- and lowoverdensity subsamples do not appear to have significantly different $\overline{\mathrm{BHAR}}$, indicating that SMBH growth does not have a strong dependence on local environment at a given $M_{\star}$. Any small apparent $\overline{\mathrm{BHAR}}$ differences between the high- and low-overdensity subsamples are likely just due to statistical fluctuations, as some blue points in Fig. 7 (left) are slightly above the corresponding red points, while other blue points are below. The subsamples' median $\overline{\mathrm{BHAR}} 1 \sigma$ uncertainty is 0.09 dex. Therefore, if the high- and low-overdensity subsamples had $\overline{\text { BHAR }}$ differing by $\gtrsim 0.09$ dex systematically, the blue and red points would be significantly separated in Fig. 7 (left). In all redshift bins, $\overline{\mathrm{BHAR}}$ rises toward the high $M_{\star}$ regime. Yang et al. (2018) have modelled the $\overline{\mathrm{BHAR}}-M_{\star}$ relations at different redshifts in detail, and our data points are consistent with their results (see Fig. 7). The $\overline{\mathrm{BHAR}}$ contributed from stacking is generally $\approx 0.5$ dex lower than the total $\overline{\mathrm{BHAR}}$. Therefore, most X-ray emission is from the X-ray-detected sources.

The two-subsample split (Fig. 7, left) guarantees that both subsamples have half the number of sources in each $M_{\star}$ bin, and thus the $\overline{\text { BHAR }}$ uncertainties are relatively small (median uncertainty $=0.09 \mathrm{dex}$ ) for the subsamples. We also probe more extreme overdensity regimes by comparing $\overline{\mathrm{BHAR}}$ of subsamples with the highest 20 per cent of overdensities and the lowest 20 per cent of overdensities (see Fig. 7, right). The high- and low-overdensity subsamples also have similar $\overline{\mathrm{BHAR}}$ in general, although the $\overline{\mathrm{BHAR}}$ uncertainties become larger (median uncertainty $=0.13 \mathrm{dex}$ ) compared to those in Fig. 7 (left) due to reduced subsample sizes. In Fig. 7, there are two pairs of high- and low-overdensity points that are separated above a $2 \sigma$ confidence level. These deviations are likely due to statistical fluctuations. There are a total of 26 pairs of points in Fig. 7 (i.e. 26 trials), and we expect to find $\lesssim 4$ such deviations (99 per cent confidence level, calculated with a binomial distribution). Also, our detailed quantitative analyses in Section 3.1.2 do not find statistically significant $\overline{\mathrm{BHAR}}$-overdensity relation when $M_{\star}$ is controlled.

\subsubsection{Partial correlation analyses}

In Section 3.1.1, we qualitatively showed that the high-overdensity subsamples have similar $\overline{\mathrm{BHAR}}$ as the corresponding lowoverdensity subsamples when controlling for $M_{\star}$. This result indicates that $\mathrm{SMBH}$ growth, at a given $M_{\star}$, does not significantly depend on local environment. We further quantitatively verify this point via partial-correlation (PCOR) analysis (e.g. Johnson \& Wichern 2002).

Following Yang et al. (2017), we utilize PCOR.R in the R statistical package to perform PCOR analyses (Kim 2015). We bin sources in the overdensity $-M_{\star}$ plane and derive $\overline{\mathrm{BHAR}}$ for each bin. The bin boundaries of overdensity and $M_{\star}$ are the same as in Section 3.1.1. Fig. 8 shows the resulting $\overline{\mathrm{BHAR}}$ on overdensity $-M_{\star}$ grids. Similar to Yang et al. (2017), we input the $\log \overline{\mathrm{BHAR}}, \log (1+\delta)$, and $\log M_{\star}$ values into PCOR.R, where the $\log (1+\delta)$ and $\log M_{\star}$ are the medians in each bin. We study the $\overline{\text { BHAR }}$-overdensity $(\overline{\text { BHAR }}-$ $M_{\star}$ ) correlation while controlling for the effects of $M_{\star}$ (overdensity) via all three statistics available in PCOR.R (Pearson, Spearman, and Kendall). The Pearson statistic assumes loglinear relations, while the Spearman and Kendall non-parametric statistics are rank-based and do not have such assumptions. 

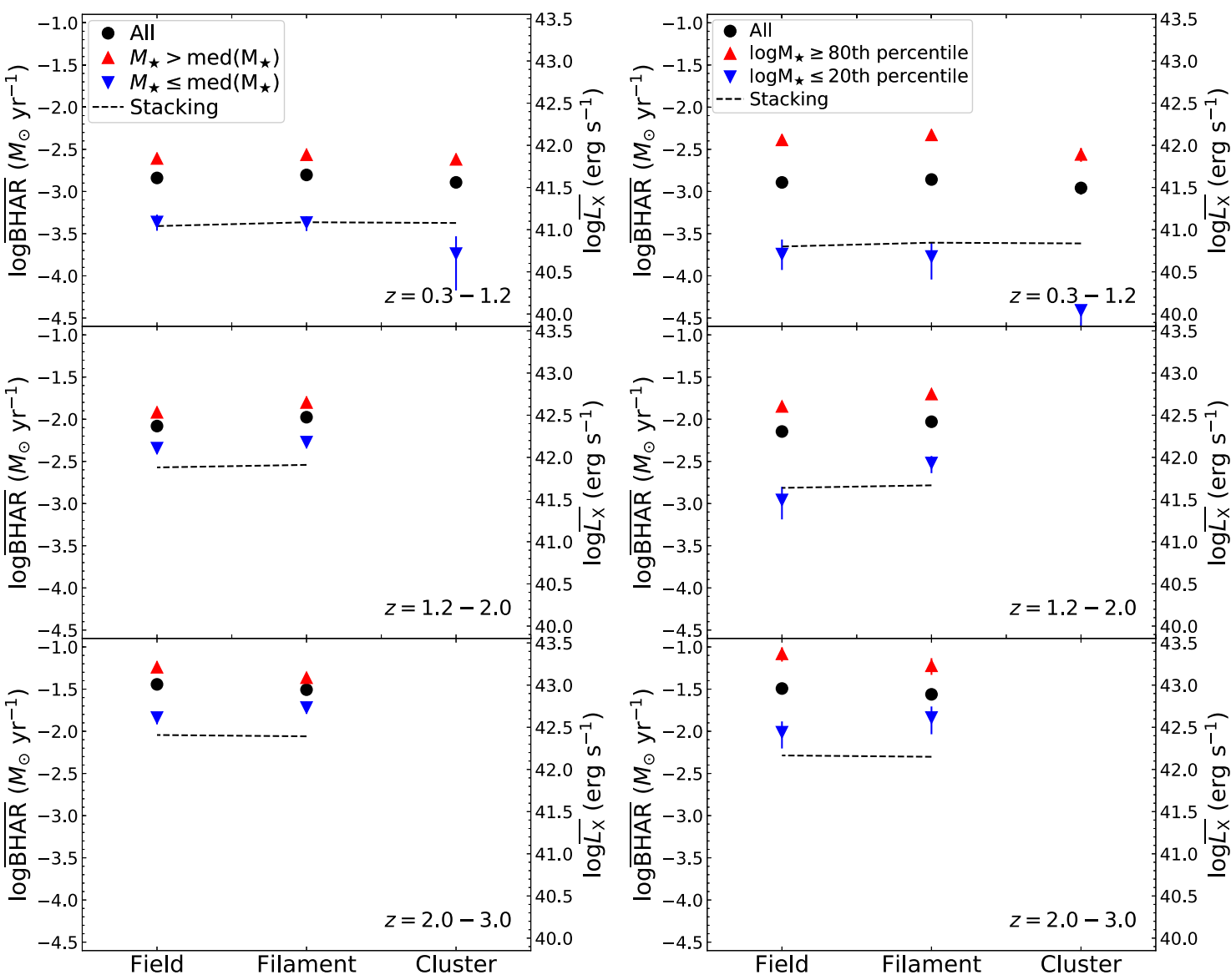

Figure 11. Same format as Fig. 6, but for $\overline{\mathrm{BHAR}}$ versus cosmic-web environment. We do not assign cluster environment at redshifts above $z=1.2$ due to its generally weak signals (see Section 2.3.2). The high $-M_{\star}$ subsamples have $\overline{\text { BHAR }}$ significantly higher than their corresponding low- $M_{\star}$ subsamples.

The results are listed in Table 3 . The $\overline{\mathrm{BHAR}}-M_{\star}$ correlation is statistically significant for all statistical techniques and across all redshift ranges. In contrast, the $\overline{\mathrm{BHAR}}$-overdensity correlation is not significant $(<3 \sigma)$ under any statistic in any redshift range. To visualize the PCOR results, we perform a least- $\chi^{2} \operatorname{loglinear}$ fit of the $\overline{\mathrm{BHAR}}-M_{\star}(\overline{\mathrm{BHAR}}-$ overdensity $)$ relation. We then fit the residual $\overline{\mathrm{BHAR}}$ as a function of overdensity $\left(M_{\star}\right)$. This procedure is similar to the Pearson statistic in PCOR analyses. The uncertainties of the best fit are estimated based on Markov Chain Monte Carlo sampling with EMCEE (Foreman-Mackey et al. 2013). Fig. 9 displays the fitting results for $z=0.3-1.2$. The best fit of the residual $\overline{\mathrm{BHAR}}$-overdensity relation is consistent with a flat model at a $3 \sigma$ confidence level, while the residual $\overline{\mathrm{BHAR}}-M_{\star}$ relation is steep. The conclusion also holds for the other two redshift ranges. In Fig. 9 (bottom), the data points at $\log M_{\star} \approx 10.75$ tend to be above the fit. This perhaps indicates a loglinear model (assumed in the Pearson statistic) is not enough to describe fully the $\overline{\mathrm{BHAR}}-M_{\star}$ relation, consistent with previous works (e.g. Georgakakis et al. 2017; Aird et al. 2018; Yang et al. 2018). The Spearman and Kendall statistics do not assume a log-linear model, and they lead to qualitatively similar results as the Pearson statistic (see Table 3). Also, we note that since there are a total of such 12 trials in Fig. 9 (considering both the top and bottom panels), it is not surprising that one such deviation happens by chance (see Section 3.1.1).

Therefore, we conclude that the $\overline{\mathrm{BHAR}}$ significantly depends on $M_{\star}$ but not overdensity. The conclusion is qualitatively supported by Figs 1, 4, and 8. In Figs 1 and 4, the X-ray-detected sources preferentially appear in the high- $M_{\star}$ regime but do not show much dependence on overdensity. In Fig. 4, X-ray sources have median overdensity values similar to those of all galaxies. In Fig. 8, the $\overline{\mathrm{BHAR}}$ gradient is strong in the $x\left(M_{\star}\right)$ direction, but weak in the $y$ (overdensity) direction.

Our analyses above are based on the $\overline{\mathrm{BHAR}}$ technique to assess $\mathrm{SMBH}$ growth. Another common technique in the literature is to consider AGN fractions above a given $L_{\mathrm{X}}$ threshold (e.g. Silverman et al. 2009; Xue et al. 2010). Compared to our $\overline{\text { BHAR }}$ approach, the AGN-fraction approach is less informative and less physical, because it needs a pre-defined $L_{\mathrm{X}}$ threshold which depends on $\mathrm{X}$ ray survey sensitivity, and it does not consider X-ray emission from undetected sources. Also, unlike the $\overline{\mathrm{BHAR}}$ approach, the AGNfraction method weights low- $L_{\mathrm{X}}$ and high- $L_{\mathrm{X}}$ AGNs equally, as long as they are above the $L_{X}$ threshold, thereby sacrificing information. However, the AGN-fraction method still serves as a common alternative way to assess SMBH accretion (e.g. Lehmer et al. 2013; Martini et al. 2013). For a consistency check, we also present the AGN fractions in different $M_{\star}$ bins for different local environments in Table 4 and Fig. 10. The AGN fractions are calculated as the fractions of X-ray-detected sources above $\log L_{X}=42.6(z=0.3$ $1.2), 43.1(z=1.2-2.0)$, and $43.5(z=2.0-3.0)$, respectively. For each redshift range, the threshold is chosen as the $\log L_{X}$ completeness limit at the redshift upper boundary (see Fig. 1). Due to the differences in $L_{X}$ thresholds, the AGN fractions (Table 4) at different redshift ranges are not directly comparable. The AGNfraction errors are derived as $1 \sigma$ binomial uncertainties using the 


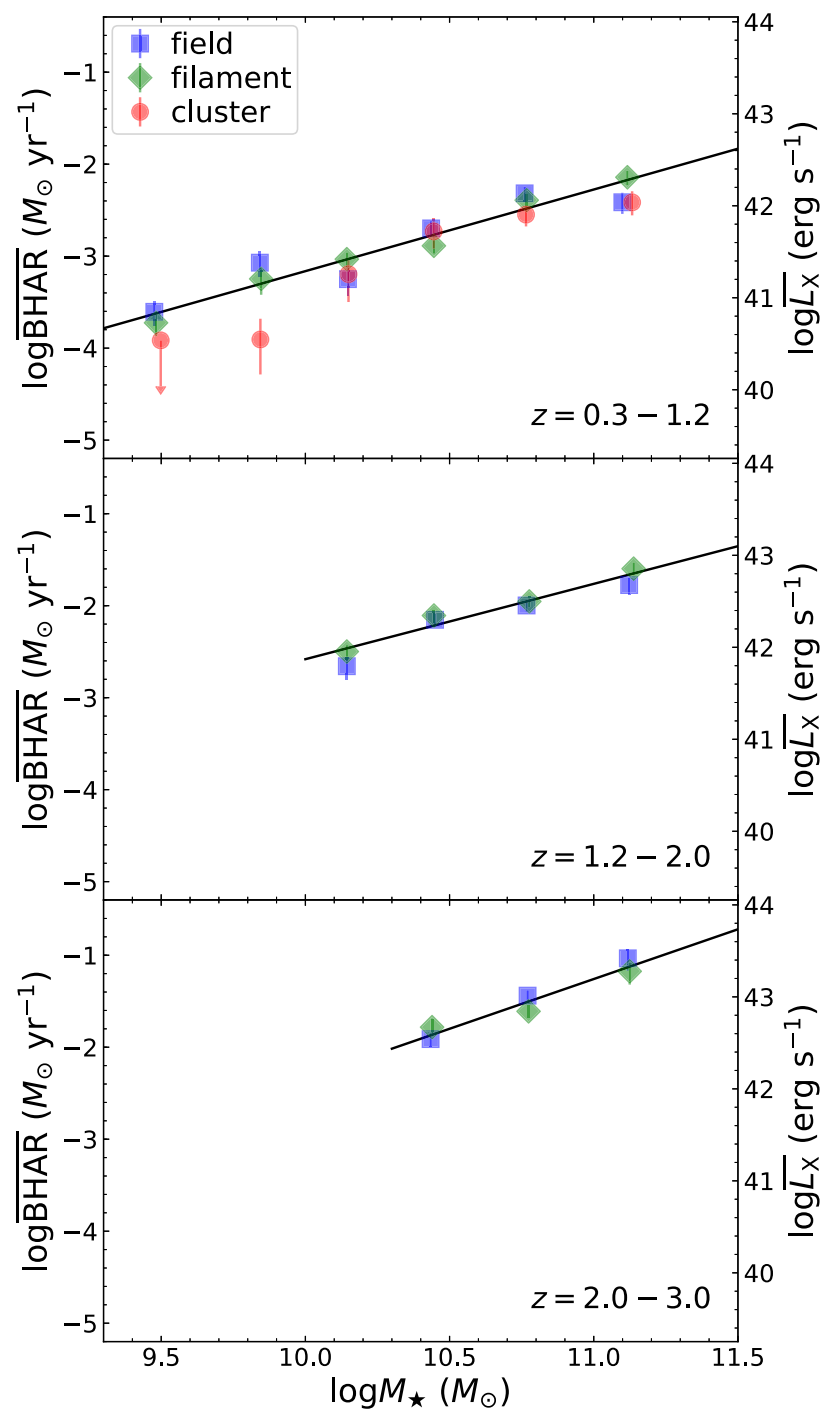

Figure 12. $\overline{\mathrm{BHAR}}$ as a function of $M_{\star}$ for different cosmic-web environments. $\overline{L_{\mathrm{X}}}$ is marked on the right-hand side of each panel. The black line represents the best-fitting loglinear model using all the data points. At a given $M_{\star}$, the $\overline{\mathrm{BHAR}}$ values are similar for different cosmic-web environments.

Table 5. Best-fitting AIC values of the $\overline{\mathrm{BHAR}}-M_{\star}$ relation (see Section 3.2).

\begin{tabular}{lrrr}
\hline Redshift & $\mathrm{AIC}_{1}$ & $\mathrm{AIC}_{2}$ & $\Delta \mathrm{AIC}$ \\
\hline $0.3-1.2$ & 32.48 & 33.35 & 0.9 \\
$1.2-2.0$ & 12.34 & 14.38 & 2.0 \\
$2.0-3.0$ & 9.82 & 9.75 & -0.1 \\
\hline
\end{tabular}

ASTROPY module 'BINOM_CONF_INTERVAL' (e.g. Cameron 2011). From Table 6 and Fig. 10, at given $M_{\star}$ and redshift, the AGN fractions are generally similar in different overdensity bins, consistent with our PCOR analyses. This consistency indicates that our conclusions are not affected by the X-ray stacking procedures (especially the masking of X-ray extended sources; see Section 2.4.2), since the AGN fractions are based on X-ray-detected sources only. Also, the AGN fraction rises toward massive galaxies, as expected from the strong $\overline{\mathrm{BHAR}}-M_{\star}$ relation (Fig. 7). In Fig. 10 (both top and middle panels), the lowest overdensity bin appears to have lower AGN fraction than other overdensity bins at $\log M_{\star} \approx 11.1$. However, the differences are not significant at a $2 \sigma$ confidence level. Also, considering there are many (78) points in Fig. 10, it is natural that a few deviations happen considering the 'number of trials' effect detailed in Section 3.1.1. Therefore, we consider the apparent differences are likely due to statistical fluctuations.

\section{2 $\overline{\text { BHAR }}$ versus cosmic-web environment}

In Section 3.1, we show that the $\overline{\mathrm{BHAR}}$ is not related to overdensity (on sub-Mpc scales) at given $M_{\star}$. In this section, we investigate the dependence of $\overline{\mathrm{BHAR}}$ on cosmic-web environment $(\sim 1-10 \mathrm{Mpc}$ scales). Following Section 3.1.1, we derive the $\overline{\mathrm{BHAR}}$ for galaxies in field, filament, and cluster environments, respectively. The cosmic web describes global environment on $\approx 1-10 \mathrm{Mpc}$ scales (Section 2.3.2). The results are displayed in Fig. 11. The $\overline{\mathrm{BHAR}}$ does not show a significant trend as a function of cosmic-web environment. For each environment bin, we further divide the sources into high $-M_{\star}$ and low- $M_{\star}$ subsamples, respectively, and calculate $\overline{\mathrm{BHAR}}$ for each subsample. Similar to Fig. 6, the high- $M_{\star}$ subsamples have significantly higher $\overline{\mathrm{BHAR}}$ than the corresponding low $-M_{\star}$ subsamples, consistent with the dominant $\overline{\mathrm{BHAR}}-M_{\star}$ relation (Section 3.1).

We also bin our samples based on $M_{\star}$, and further divide each bin into subsamples for different parts of the cosmic web. We calculate $\overline{\mathrm{BHAR}}$ for each subsample and show the results in Fig. 12. The $\overline{\mathrm{BHAR}}$ values are not systematically different for different cosmicweb environments.

Now we test the $\overline{\mathrm{BHAR}}$ dependence on cosmic-web environment quantitatively. Unlike in Section 3.1.2, we do not perform a PCOR analysis, because the cosmic-web environment (field, filament, and cluster) is not a continuous quantity. Instead, we employ another statistical analysis based on the Akaike information criterion (AIC; Akaike 1974). The AIC is designed for model selection and defined as $\mathrm{AIC}=C+2 k$, where $C$ is the fitting statistic $\left(\chi^{2}\right.$ for least-squares fitting) and $k$ is the number of free parameters in the model. If one model has an AIC value much smaller than another model $(\triangle \mathrm{AIC}<$ $\Delta \mathrm{AIC}_{\text {thresh }}$ ), then the former is considered superior to the latter (see e.g. section 2.6 of Burnham \& Anderson 2002). In our analyses, we choose $\Delta \mathrm{AIC}_{\text {thresh }}=-7$, corresponding to a $3 \sigma$ confidence level under the situation where the model parameter uncertainties are Gaussian (e.g. Murtaugh 2014).

We apply the AIC technique to our data points in Fig. 12. For each redshift range, we perform a least- $\chi^{2}$ fit to all the data points with a loglinear model, $\log \overline{\mathrm{BHAR}}=A \times \log M_{\star}+B$, where $A$ and $B$ are free model parameters. ${ }^{4}$ We calculate the AIC value $\left(\mathrm{AIC}_{1}\right)$ for this fitting. The best-fitting models are displayed in Fig. 12. We then create a set of three independent loglinear models, i.e. $\log \overline{\mathrm{BHAR}}=$ $A_{\text {field }} \times \log M_{\star}+B_{\text {field }}, \log \overline{\mathrm{BHAR}}=A_{\text {filament }} \times \log M_{\star}+B_{\text {filament }}$, and $\log \overline{\mathrm{BHAR}}=A_{\text {cluster }} \times \log M_{\star}+B_{\text {cluster }}$, to fit the data. ${ }^{5}$ As the subscripts indicate, each model is used to fit the data points of the corresponding cosmic-web environment in Fig. 12. We derive the $\mathrm{AIC}$ value $\left(\mathrm{AIC}_{2}\right)$ for this multimodel fitting. If $\triangle \mathrm{AIC}=\mathrm{AIC}_{2}-$ $\mathrm{AIC}_{1}<-7$, then the $\overline{\mathrm{BHAR}}-M_{\star}$ relations might be different for different cosmic-web environments. The resulting AIC values are listed in Table 5. For all three redshift ranges, the $\triangle \mathrm{AIC}$ values

\footnotetext{
${ }^{4}$ The single upper limit point in Fig. 12 is not used in the fitting.

${ }^{5}$ For $z>1.2$, we only create the field and filament models, as we do not assign cluster environment (see Section 2.3.2).
} 
Table 6. AGN fractions (per cent) for different cosmic-web environments.

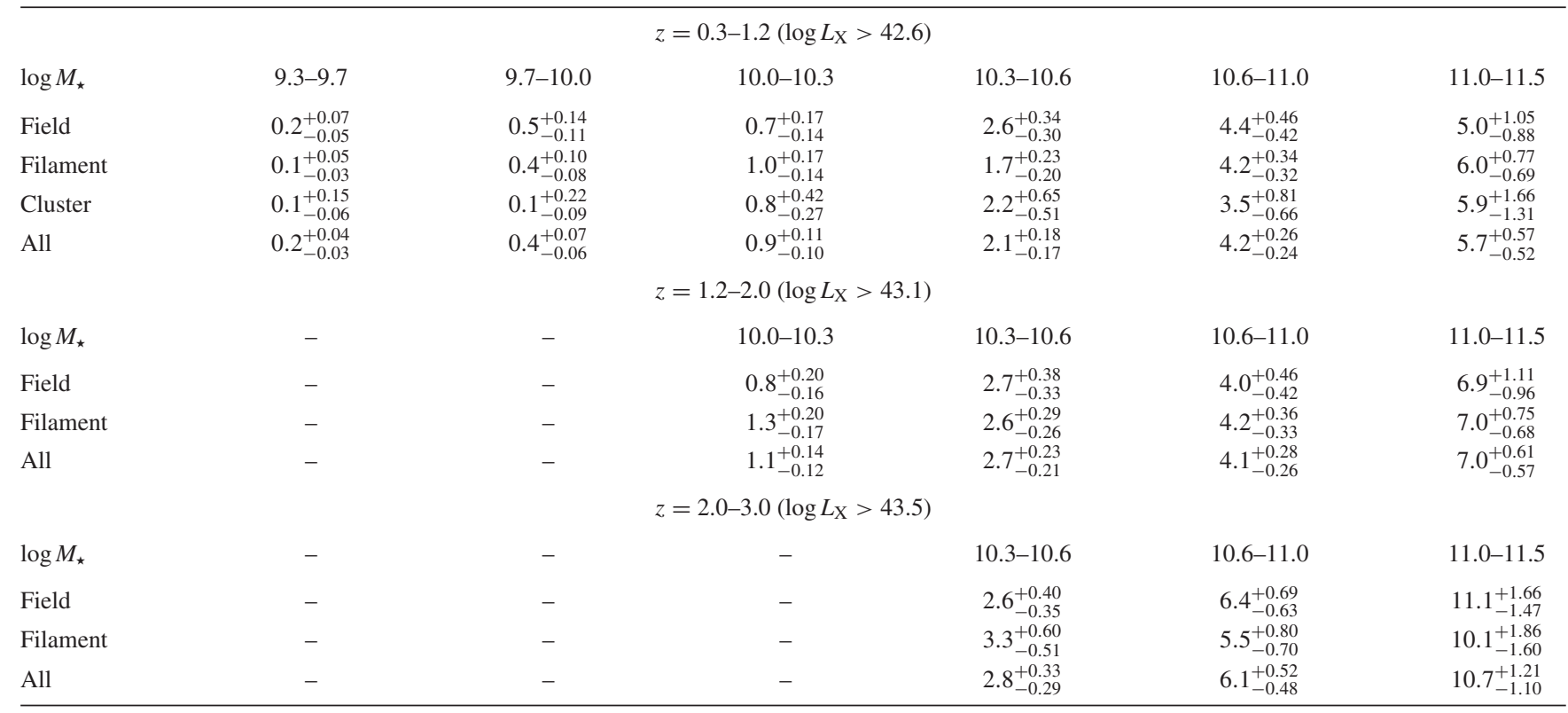

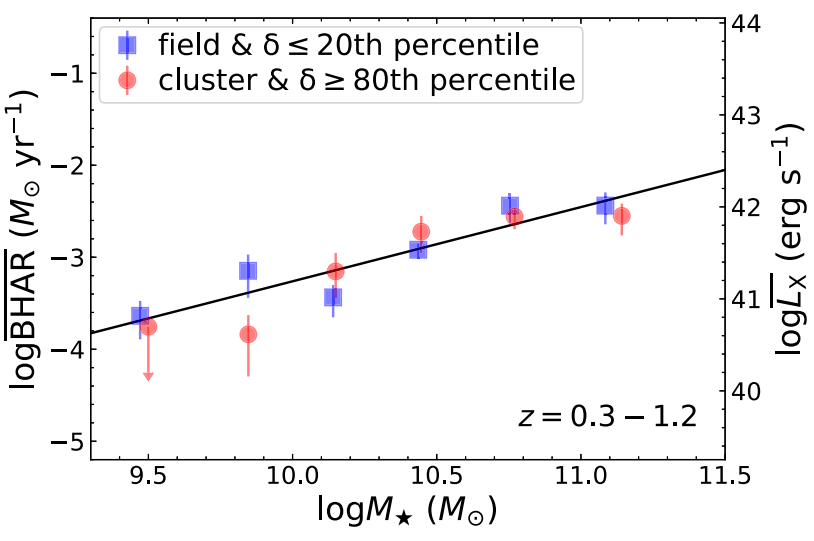

Figure 13. Same format as Fig. 12 (top), but for cluster versus field environments. Here, we limit the cluster (field) galaxies to those with the highest (lowest) 20 per cent overdensity in each $M_{\star}$ bin. At a given $M_{\star}$, the $\overline{\mathrm{BHAR}}$ values are similar for cluster and field environments.

are above -7 . Therefore, the differences among the $\overline{\mathrm{BHAR}}-M_{\star}$ relations for different cosmic-web environments are not statistically significant. However, the non-detection of a $\overline{\mathrm{BHAR}}-\mathrm{environment}$ correlation might be, in principle, due to the limited sensitivity of our data. Martini et al. (2009) found that the AGN fraction in rich clusters is $\approx 0.7$ dex below that in the field at $z \lesssim 1$. A natural question is whether our data are sensitive enough to detect such $\overline{\mathrm{BHAR}}$ differences, i.e. $\overline{\mathrm{BHAR}}$ drops by 0.7 dex from the field to cluster environments. To answer this question, we perform a test. For our $z=0.3-1.2$ bin, we systematically shift our cluster (field) $\overline{\mathrm{BHAR}}$ by $-0.35 \mathrm{dex}(+0.35 \mathrm{dex})$ and recalculate $\triangle \mathrm{AIC}$. We find $\triangle \mathrm{AIC}=-114$, much lower than our threshold $(-7)$. Therefore, if our $\overline{\mathrm{BHAR}}$ dropped by 0.7 dex from the field to cluster environments, we would definitely detect the environmental dependence of $\overline{\mathrm{BHAR}}$. In fact, we find that our data are sensitive at a $\approx 3 \sigma$ level to $\mathrm{a} \approx 0.2$ dex difference of $\overline{\mathrm{BHAR}}$ from the field to cluster environments at $z=0.3-1.2$. This difference between our work and Martini et al. (2009) might be due to the lack of rich clusters in our sample (see Section 4.1 for more discussion).

In Fig. 13, we also compare $\overline{\mathrm{BHAR}}$ for cluster and field environments at $z=0.3-1.2$. Here, we limit the cluster (field) galaxies to those with the highest (lowest) 20 per cent overdensity in each $M_{\star}$ bin. ${ }^{6}$ In this way, we probe the most-extreme environments. We perform AIC analyses and obtain $\triangle \mathrm{AIC}=3.9$, above the threshold $(-7)$. Therefore, the $\overline{\mathrm{BHAR}}-M_{\star}$ relations for these two extreme environments are also not statistically different. At higher redshift, we have also performed similar analyses for filament versus field environments and reached the same conclusion.

As in Section 3.1.2, we also calculate AGN fractions for different cosmic-web environments for a consistency check. The results are presented in Table 6 and Fig. 14. The AGN fractions are generally similar for different cosmic-web environments when controlling for $M_{\star}$, consistent with our AIC analyses. Our AGN fractions for $\log M_{\star}>10.3$ are $\approx 2$ per cent -6 per cent at $z=0.3-1.2$. This range is consistent with the results of Silverman et al. (2009, see their table 2), who found an AGN fraction of $\approx 3$ per cent for $\log M_{\star}>$ 10.4 at $z<1$, independent of environment. Our AGN fractions for $\log M_{\star}=11-11.5$ at $z=0.3-1.2$ are $\approx 6$ per cent, similar to that derived for SDSS galaxies of similar $M_{\star}$ at $z \approx 0.5$ (Haggard et al. 2010).

\subsection{Tests in narrower redshift bins}

Our analyses above adopt relatively wide redshift bins, i.e. $z=0.3-$ $1.2,1.2-2.0$, and $2.0-3.0$, to retain relatively large sample size in each bin. Considering that both galaxy and AGN properties as well as cosmic environment evolve with redshift, the $\overline{\mathrm{BHAR}}-$ environment relation might also have redshift dependence. To test for possible redshift dependence, we repeat our analyses in Sections 3.1 and 3.2 using narrower redshift bins, i.e. $z=0.3-0.8$,

\footnotetext{
${ }^{6} \mathrm{Here}$, we do not limit cluster galaxies to those also identified by George et al. (2011), because this would lead to too few sources (only $<10$ AGNs) for our analyses, as the cluster-member catalogue in George et al. (2011) is not complete (see Section 2.3.2).
} 


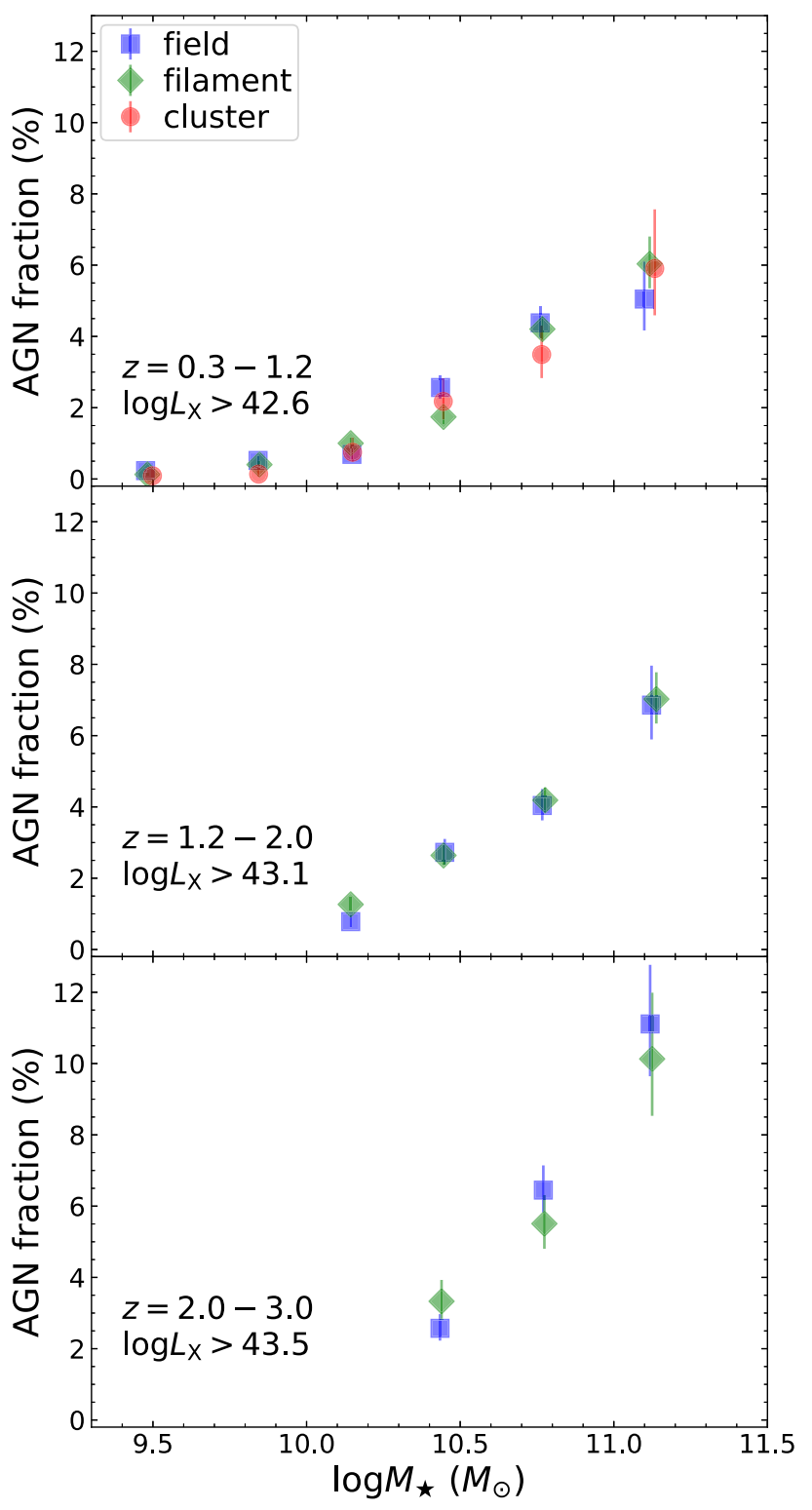

Figure 14. AGN fraction as a function $M_{\star}$ for different cosmic-web environments. The data are from Table 6 . At a given $M_{\star}$, AGN fractions are similar for different cosmic-web environments.

$0.8-1.2,1.2-1.6,1.6-2.0,2.0-2.5$, and 2.5-3.0. This procedure reduces the sample size in each bin and thus increases the uncertainties on $\overline{\mathrm{BHAR}}$ in general. In the new analyses with narrower redshift bins, we still do not find any significant $\overline{\mathrm{BHAR}}$ dependence on either overdensity or cosmic-web environment, consistent with the results in Sections 3.1 and 3.2. Fig. 15 shows some example figures for $z=0.8-1.2$, and the figures for other narrower redshift bins are qualitatively similar. Therefore, our main conclusions are unlikely to be affected by our choice of relatively wide redshift bins.

\section{DISCUSSION}

We discuss the physical implications of our results in Section 4.1. We compare our results with previous observations of BHARenvironment relations in Section 4.2.
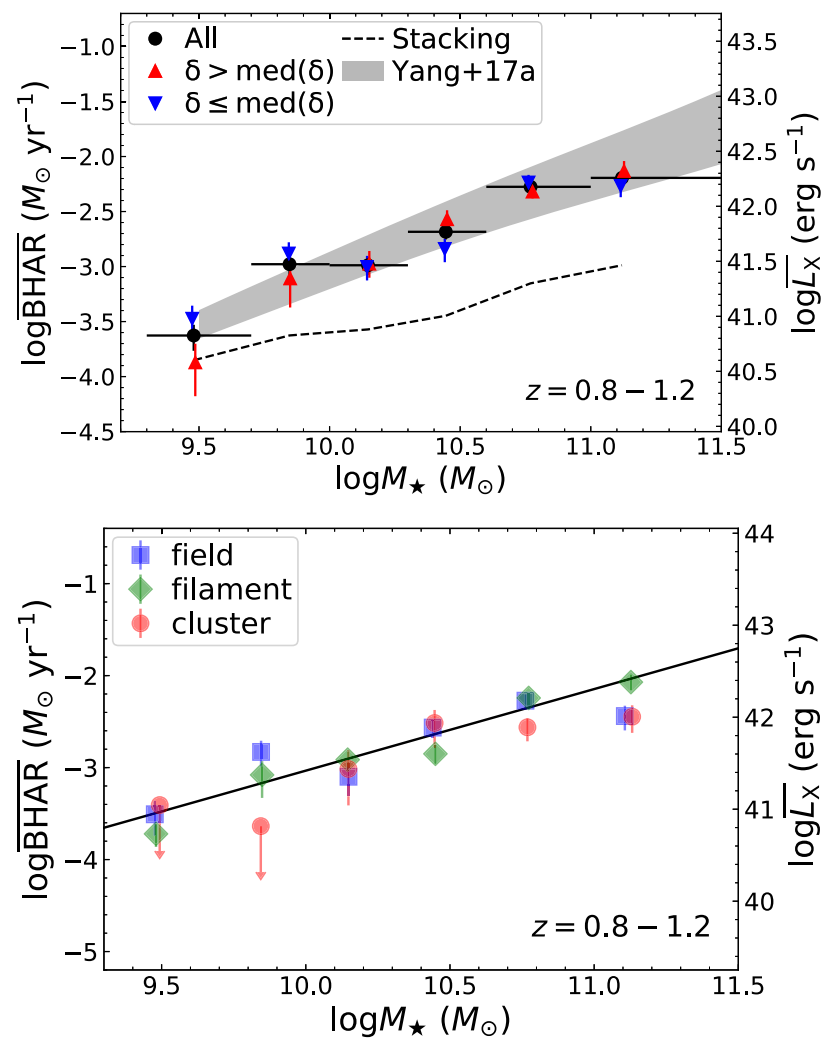

Figure 15. The top and bottom panels follow the same formats as Figs 7 and 12, but for the narrower redshift bin of $z=0.8-1.2$. Still, we do not find significant $\overline{\mathrm{BHAR}}$ dependence on environment. This conclusion also applies for other narrower redshift bins in Section 3.3.

\subsection{Physical implications}

Our results indicate that SMBH accretion is fundamentally related to $M_{\star}$. At a given $M_{\star}$, our $\overline{\mathrm{BHAR}}$ does not show significant dependence on host-galaxy environment. Since galaxy environment is largely determined by dark matter, which generally dominates the gravitational field on $\gtrsim$ sub-Mpc scales, our conclusions suggest SMBH growth is primarily related to baryons rather than dark matter (e.g. Kormendy \& Ho 2013; Yang et al. 2018). The broad physical picture is likely that dark-matter density fluctuations lead to the formation of haloes, allowing baryons to condense into the halo centres and form galaxies. The galaxies feed their SMBHs with cold gas via baryonic physics, e.g. disc instabilities and galaxy bars (e.g. Alexander \& Hickox 2012, and references therein). This scenario indicates that, in future studies of SMBH-galaxy co-evolution, it is critical to focus on relations between BHAR and host-galaxy intrinsic properties (e.g. $M_{\star}, \mathrm{SFR}$, and morphology) rather than the environment. Small but deep surveys such as the CDF (e.g. Xue et al. 2016; Luo et al. 2017) and CANDELS (Grogin et al. 2011; Koekemoer et al. 2011) are ideal for studying SMBH-galaxy coevolution.

It is well established that environment affects galaxy evolution. At a given $M_{\star}$ and at $z \lesssim 1$, the quiescent-galaxy fraction (as defined in Section 2.2) rises toward high-density regions, and this effect is often termed 'environmental quenching' (e.g. Peng et al. 2010; Scoville et al. 2013; Darvish et al. 2015, 2016, 2017; Laigle et al. 2018). Fig. 16 shows the quiescent-galaxy fraction in our sample as a function of $M_{\star}$ for different cosmic-web environments (see Section 2.2 for star-forming/quiescent classifications). At $z=0.3-$ 


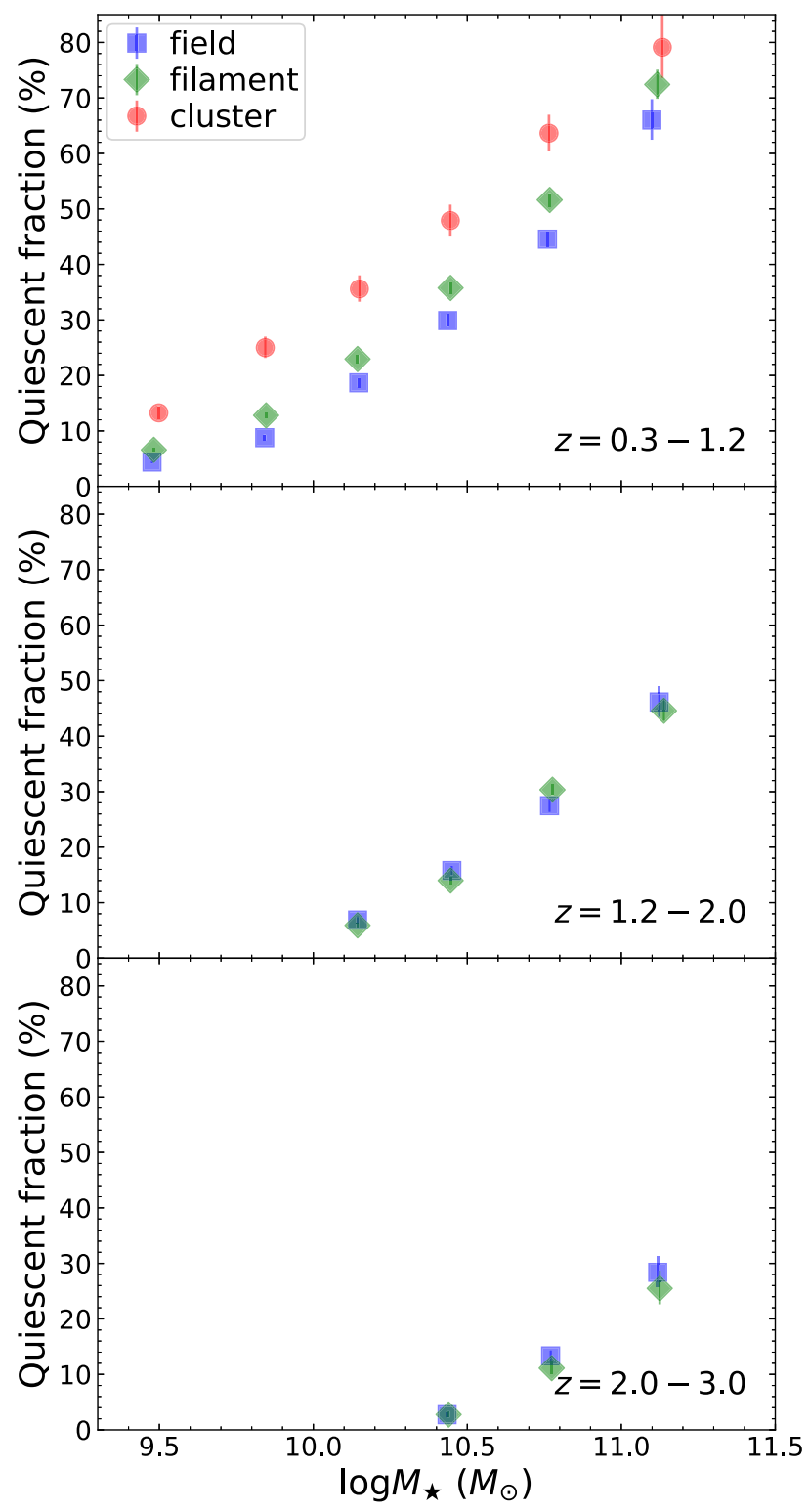

Figure 16. Quiescent-galaxy fraction versus stellar mass for different cosmic-web environments. The star-forming/quiescent classifications are based on a standard colour-colour scheme (see Section 2.2). At $z=0.3-1.2$ and a given $M_{\star}$, the quiescent-galaxy fraction rises from the field to cluster environments (environmental quenching). At $z>1.2$, galaxies associated with the field and filament environments have similar quiescent-galaxy fractions.

Table 7. Quiescent-galaxy fractions (per cent) for AGN host galaxies.

\begin{tabular}{ccccc}
\hline Redshift & Field & Filament & Cluster & All \\
\hline $0.3-1.2$ & $18.8_{-2.40}^{+2.66}$ & $26.5_{-2.25}^{+2.38}$ & $29.3_{-5.59}^{+6.29}$ & $24.0_{-1.62}^{+1.70}$ \\
$1.2-2.0$ & $13.9_{-2.26}^{+2.62}$ & $10.0_{-1.45}^{+1.67}$ & - & $11.4_{-1.26}^{+1.40}$ \\
$2.0-3.0$ & $7.6_{-1.73}^{+2.19}$ & $6.6_{-1.92}^{+2.63}$ & - & $7.2_{-1.35}^{+1.63}$ \\
\hline
\end{tabular}

1.2 and a given $M_{\star}$, the quiescent-galaxy fraction significantly rises from the field to cluster environments; at higher redshifts, this environmental dependence seems to disappear. Therefore, environmental quenching at $z=0.3-1.2$ is significantly observed in our sample. For a given cosmic-web environment, the quiescent-galaxy fraction also rises toward high $M_{\star}$ at all redshifts. This $M_{\star}$ dependence is expected from previous studies (e.g. Brammer et al. 2011; Davidzon et al. 2017).

In contrast, our $\overline{\mathrm{BHAR}}$ does not show a significant dependence on environment, if $M_{\star}$ is controlled (see Section 3). These different behaviours of SMBH accretion and star formation suggest that SMBH and galaxy stellar-mass growth are not strongly coupled in general (e.g. Yang et al. 2017, 2018), although we cannot rule out a weak secondary $\overline{\text { BHAR}}-$ SFR relation (see Section 4.2). The potential physical mechanisms responsible for environmental quenching such as tidal interaction and ram-pressure stripping (Section 1) might only have limited effects on SMBH accretion.

Since galaxy evolution has significant dependence on environment at low redshift $(z \lesssim 1)$, the host-galaxy types of AGNs might also depend on environment. In Table 7, we list the quiescent-galaxy fractions for AGNs (as defined in Section 3.1.2) in different cosmicweb environments. At $z=0.3-1.2$, the quiescent-galaxy fraction of AGN hosts appears to rise from the field to clusters, similar to the trend for normal galaxies (see Fig. 16). At higher redshift, if anything, the trend seems to be the opposite going from the field to filament environments. However, these trends are not statistically significant at a $3 \sigma$ confidence level due to our limited AGN sample size. Future work with much larger AGN samples can determine these trends more accurately.

\subsection{Previous works on BHAR versus environment}

Based on sources at $z \lesssim 1$, observations have found that cluster $\left(M_{\text {halo }} \lesssim 10^{14} \mathrm{M}_{\odot}\right)$ and field environments have similar X-ray AGN fractions among massive galaxies, consistent with our results (e.g. Georgakakis et al. 2008; Silverman et al. 2009; Koulouridis et al. 2014). However, these analyses are often restricted to low redshift relatively bright galaxies. Thanks to the reliable photo- $z$ measurements and our improved methodology for assessing SMBH accretion, our work is able to investigate $\overline{\mathrm{BHAR}}$-environment relations for all galaxies above the $M_{\star}$ completeness limits up to $z=3$. Importantly, our study covers $z \approx 1.5-2.5$ where cosmic AGN activity peaks.

Some studies find that, at $z \lesssim 1$, the X-ray AGN fractions in rich clusters $\left(M_{\text {halo }} \sim 10^{15} \mathrm{M}_{\odot}\right)$ are generally lower than those in the field (e.g. Martini et al. 2009; Ehlert et al. 2014). Due to the lack of excellent multiwavelength coverage for $M_{\star}$ calculation, these studies often adopt a simple $R$-band magnitude cut to approximate an $M_{\star}$ cut of the galaxy population (e.g. Cappellari 2016). However, we note that consensus has not been widely reached on whether rich clusters have lower AGN fractions than the field. For example, Haggard et al. (2010) found that rich clusters and the field have similar AGN fractions at $z=0.05-0.31$, when the same magnitude and $L_{\mathrm{X}}$ cuts are applied to the cluster and field populations. If AGN activity is indeed suppressed in rich clusters at a given $M_{\star}$, the physical reason might be different galaxy types in cluster and field environments. Rich clusters, especially in their central regions, are dominated by the quiescent-galaxy population, which tends to have lower AGN fractions than the star-forming population at a given $M_{\star}$ (e.g. Wang et al. 2017; Aird et al. 2018; Yang et al. 2018). We cannot study such rich clusters in our work, because they are rare and generally absent in COSMOS, where the clusters 
typically have $M_{\text {halo }} \lesssim 10^{14} \mathrm{M}_{\odot}$ (e.g. Knobel et al. 2009; George et al. 2011). However, only a small fraction of the galaxy population ( $\lesssim 1$ per cent) lives in rare rich clusters with $M_{\text {halo }} \sim 10^{15} \mathrm{M}_{\odot}$ (e.g. table 1 of Bahcall 1999). Our work covers a large comoving volume $\left(\approx 10^{7} \mathrm{Mpc}^{3}\right.$ for each redshift bin; Section 2.1$)$, and thus we probe the main range of cosmic environments in the overall Universe with $M_{\text {halo }} \approx 10^{11}-10^{14} \mathrm{M}_{\odot}$. We do not find significant $\overline{\mathrm{BHAR}}-$ environment relations when controlling for $M_{\star}$, even at the extremes of the environments we sample (e.g. Figs 7 and 13). Therefore, we conclude that, for the overall galaxy population, $\overline{\mathrm{BHAR}}$ generally does not depend on cosmic environment once $M_{\star}$ is controlled, although this conclusion might not hold for galaxies living in rare rich clusters.

At high redshift $(z \gtrsim 2)$, observations suggest that some massive protoclusters might have elevated AGN activity (see Section 1; but also see Macuga et al. 2018). For example, SSA 22 is a prominent protocluster at $z \approx 3.1$, and it is likely a progenitor of local rich clusters with $M_{\text {halo }} \sim 10^{15} \mathrm{M}_{\odot}$ (e.g. Steidel et al. 1998). Lehmer et al. (2009) estimated that its X-ray AGN fraction is $\approx 5$ per cent-10 per cent among Lyman break galaxies (LBGs) and Ly $\alpha$ emitters (LAEs), considerably higher than the AGN fraction $(\approx 1$ per cent -2 per cent $)$ among LBGs and LAEs in the field at similar redshifts. However, the LBGs and LAEs in SSA 22 might have different $M_{\star}$ compared to the LBGs and LAEs in the field, and the difference in $M_{\star}$ could drive the apparent differences in AGN fraction (see Table 6). Also, the SSA 22 AGN sample size is limited $(\approx 10)$ and suffers from significant statistical uncertainty. SSA 22 has $\gtrsim 150$ galaxies in its central region of $\approx 10 \mathrm{Mpc}^{2}$ (e.g. Topping, Shapley \& Steidel 2016), translating to an overdensity value of $\log (1+\delta) \gtrsim 1.3$ (Section 2.3.1). We do not have such prominent structures in our sample at $z \gtrsim 2.5$ (Fig. 1) and thus cannot probe their AGN activity using COSMOS. Note that the estimated overdensity value of SSA 22 is for high redshifts $(z \gtrsim 2.5)$. It is not directly comparable to our structures at low redshift, since the normalization factors $\Sigma_{\text {median }}$ are different at different redshifts (see equation 1).

AGN-clustering studies also make use of source spatial distributions to probe SMBH-galaxy co-evolution. Early observations found that X-ray AGNs and normal galaxies appear to have different clustering properties (especially on $\lesssim 1 \mathrm{Mpc} h^{-1}$ comoving scales), suggesting different environmental effects for central versus satellite galaxies (e.g. Hickox et al. 2009; Miyaji et al. 2011; Allevato et al. 2012). However, galaxy properties (especially $M_{\star}$ ) were not carefully controlled among these studies. Considering the strong $\overline{\mathrm{BHAR}}-M_{\star}$ correlation (e.g. Yang et al. 2017, 2018) and the well-established relation between galaxy clustering and $M_{\star}$ (e.g. Coil et al. 2006, 2017), it is critical to match $M_{\star}$ in AGN versus galaxy clustering analyses. More recent observations show that the clustering properties of AGNs and galaxies are similar over a wide range of comoving scale $\left(\approx 0.1-30 \mathrm{Mpc} h^{-1}\right)$ when $M_{\star}$ is carefully matched, indicating that $M_{\star}$ (rather than a central/satellite effect) mainly drives the observed clustering properties of AGNs (e.g. Georgakakis et al. 2014; Leauthaud et al. 2015; Mendez et al. 2016; Powell et al. 2018). Also, our analyses do not support different environmental effects for central versus satellite galaxies, although we do not perform a central/satellite classification (Section 2.3). If, for example, environment only affects AGN activity in central galaxies, we would witness an increasingly strong $\overline{\mathrm{BHAR}}$-environment relation in more massive galaxies which have a higher chance of being central (e.g. Reddick et al. 2013). However, we do not find any significant $\overline{\mathrm{BHAR}}$-environment correlation over a wide range of stellar mass $\left(\log M_{\star} \approx 9.5-11.5\right.$; Section 3$)$. Clustering analyses infer that AGNs typically have $\log M_{\text {halo }} \approx 13$ (e.g. Allevato et al. 2011, 2014, 2016; Richardson et al. 2013). This is as expected, since low-mass haloes $\left(\log M_{\text {halo }} \lesssim 12\right)$ only host low-mass galaxies with weak $\overline{\mathrm{BHAR}}$ and high-mass haloes $\left(\log M_{\text {halo }} \gtrsim 14\right)$ are rare.

\section{SUMMARY AND FUTURE WORK}

We have studied the $\overline{\mathrm{BHAR}}$ dependence on $M_{\star}$ and environment in redshift bins of $z=0.3-1.2,1.2-2.0$, and 2.0-3.0, based on sources in the COSMOS field. Our main procedures and results are summarized below:

(i) We have compiled a large galaxy sample in the COSMOS field $(\approx 170000$ sources; Section 2.1$)$ and estimated their $M_{\star}$ via SED fitting (Section 2.2). We have measured surface overdensity (subMpc scales) and cosmic-web environment ( $\approx 1-10$ Mpc scales $)$ for our sources (Section 2.3).

(ii) We have derived $\overline{\mathrm{BHAR}}$ for different samples, considering both X-ray-detected and undetected sources (Section 2.4). For Xray-detected sources, we adopt, in order of priority, hard-, full, and soft-band fluxes, in our calculations (Section 2.4.1). This choice is to minimize the effects of X-ray obscuration. We include the X-ray emission from X-ray undetected sources via stacking (Section 2.4.2).

(iii) We do not find a statistically significant $\overline{\mathrm{BHAR}}$ dependence on overdensity or cosmic-web environment $(\approx 1-10 \mathrm{Mpc})$ for $M_{\star}$ controlled samples (Section 3 ). Instead, $\overline{\mathrm{BHAR}}$ is always strongly related to $M_{\star}$, regardless of environment. These results suggest that $\overline{\mathrm{BHAR}}$ might be primarily related to the host galaxies rather than cosmic environment on scales of $\approx 0.1-10 \mathrm{Mpc}$, which is determined by dark matter (Section 4.1). Thanks to the large comoving volume sampled $\left(\approx 10^{7} \mathrm{Mpc}^{3}\right.$ for each redshift bin), we can probe the main range of cosmic environments in the overall Universe (Section 4.2). Therefore, we conclude that, for the overall galaxy population, $\overline{\text { BHAR }}$ generally does not depend on cosmic environment once $M_{\star}$ is controlled, although this conclusion might not hold for the $\lesssim 1$ per cent of galaxies living in rare rich clusters with $M_{\text {halo }} \sim 10^{15} \mathrm{M}_{\odot}$.

(iv) In contrast to SMBH accretion, star formation activity significantly depends on environment at $z \lesssim 1$ (Section 4.1). For our sample, the quiescent-galaxy fraction rises from the field to cluster environment for $M_{\star}$-controlled samples at $z=0.3-1.2$, consistent with previous observations. The different behaviours of SMBH accretion and star formation suggest that $\mathrm{SMBH}$ and galaxy growth are not strongly coupled in general. Environment-related mechanisms such as tidal interaction and ram-pressure stripping that could shape galaxy evolution do not appear to strongly affect SMBH growth.

Future work can probe the $\overline{\mathrm{BHAR}}$ dependence on environment for larger physical scales $(\approx 10-100 \mathrm{Mpc})$. Since COSMOS alone cannot sample the full range of cosmic environments on these scales (e.g. Meneux et al. 2009; Skibba et al. 2014), these studies will need several COSMOS-like fields, e.g. XMM-LSS (Chen et al. 2018), Wide-CDF-S, and ELAIS-S1, or much larger fields such as Stripe 82 (LaMassa et al. 2013) and XMM-XXL (Pierre et al. 2016). Such larger fields can also be used to probe SMBH growth in rare rich clusters/protoclusters (see Section 4.2), while controlling for hostgalaxy properties, especially $M_{\star}$. In addition, future work may study $\overline{\text { BHAR }}$ in galaxy close pairs on $\approx 10-100 \mathrm{kpc}$ scales (e.g. Mundy et al. 2017).

The upcoming eROSITA all-sky X-ray survey will yield a sample of $\sim 10^{6}$ AGNs at $z \lesssim 1$ (e.g. Merloni et al. 2012), allowing 
studies of $\overline{\mathrm{BHAR}}$-environment relations at low-to-moderate redshift with overwhelming source statistics. In this work, we do not find significant environmental dependence of average BHAR. It is still possible that the full distribution of BHAR depends on environment, although this would require 'finely tuned' BHAR distributions in different environments to maintain constant $\overline{\mathrm{BHAR}}$. A full characterization of the BHAR distribution as a function of $M_{\star}$, environment, and redshift (e.g. Georgakakis et al. 2017; Aird et al. 2018; Yang et al. 2018) requires future X-ray observatories like Athena and Lynx, which are necessary to sample the faint end of the BHAR distribution in COSMOS-like (or larger) fields (e.g. Georgakakis 2018).

With the advance of environment-measurement methodology, new environmental metrics other than overdensity (and the consequent field/filament/cluster classification) may be developed. Future work can study the $\overline{\mathrm{BHAR}}$ dependence on these new environmental metrics (e.g. mass density instead of number density as used in this work). Future spectroscopic observations with Extremely Large Telescopes should improve the spec- $z$ completeness for COSMOS and other fields by a large factor, allowing environmental measurements with superior accuracy (e.g. reducing the projection distance from $\approx 100$ to $\approx 10 \mathrm{Mpc}$; Appendix). Based on such new spec- $z$ data, studies can revisit the $\overline{\mathrm{BHAR}}-$ environment $-M_{\star}$ connection, even for central/satellite galaxies separately.

\section{ACKNOWLEDGEMENTS}

We thank the referee for helpful feedback that improved this work. We thank Francesca Civano, Clotilde Laigle, and Mara Salvato for providing relevant data. We thank Robin Ciardullo, Antonis Georgakakis, Ryan Hickox, Donghui Jeong, Paul Martini, Qingling Ni, John Silverman, Michael Strauss, and Rosemary Wyse for helpful discussions. GY, WNB, CTC, and FV acknowledge support from Chandra X-ray Center grants GO4-15130A and AR8-19016X, NASA grant NNX17AF07G, and the NASA Astrophysics Data Analysis Program (ADAP). BD acknowledges financial support from NASA through the ADAP, grant number NNX12AE20G, and the National Science Foundation, grant number 1716907. DMA acknowledges the Science and Technology Facilities Council through grant ST/P000541/1. FEB acknowledges support from CONICYTChile (Basal-CATA PFB-06/2007, FONDO ALMA 31160033) and the Ministry of Economy, Development, and Tourism's Millennium Science Initiative through grant IC120009, awarded to The Millennium Institute of Astrophysics, MAS.

This project uses ASTROPY (a PYTHON package; see Astropy Collaboration et al. 2013, 2018) and the SVO Filter Profile Service (http://svo2.cab.intacsic.es/theory/fps/).

\section{REFERENCES}

Aird J., Coil A. L., Georgakakis A., 2017, MNRAS, 465, 3390

Aird J., Coil A. L., Georgakakis A., 2018, MNRAS, 474, 1225

Akaike H., 1974, IEEE Trans. Autom. Control, 19, 716

Alexander D. M., Hickox R. C., 2012, New Astron. Rev., 56, 93

Alexander D. M. et al., 2016, MNRAS, 461, 2944

Allevato V. et al., 2011, ApJ, 736, 99

Allevato V. et al., 2012, ApJ, 758, 47

Allevato V. et al., 2014, ApJ, 796, 4

Allevato V. et al., 2016, ApJ, 832, 70

Aragón-Calvo M. A., Jones B. J. T., van de Weygaert R., van der Hulst J. M., 2007, A\&A, 474, 315

Aragón-Calvo M. A., van de Weygaert R., Jones B. J. T., 2010, MNRAS, 408,2163
Astropy Collaboration et al., 2013, A\&A, 558, A33

Astropy Collaboration et al., 2018, preprint (arXiv:1801.02634)

Bañados E., Venemans B., Walter F., Kurk J., Overzier R., Ouchi M., 2013 , ApJ, 773, 178

Bahcall N. A., 1999, in Dekel A., Ostriker J. P., eds, Formation of Structure in the Universe, Vol. 4. Cambridge University Press, Cambridge, p. 135

Balmaverde B. et al., 2017, A\&A, 606, A23

Balogh M. et al., 2004, MNRAS, 348, 1355

Brammer G. B. et al., 2011, ApJ, 739, 24

Brandt W. N., Alexander D. M., 2015, A\&AR, 23, 1

Burnham K., Anderson D., 2002, Model Selection and Multimodel Inference: A Practical Information-Theoretic Approach, 2. Springer-Verlag, New York, p. 49

Calzetti D., Armus L., Bohlin R. C., Kinney A. L., Koornneef J., StorchiBergmann T., 2000, ApJ, 533, 682

Cameron E., 2011, PASA, 28, 128

Cappellari M., 2016, ARA\&A, 54, 597

Cautun M., Frenk C. S., van de Weygaert R., Hellwing W. A., Jones B. J. T., 2014, MNRAS, 445, 2049

Chabrier G., 2003, ApJ, 586, L133

Chen C.-T. J. et al., 2013, ApJ, 773, 3

Chen C.-T. J. et al., 2018, MNRAS, 478, 2132

Ciesla L. et al., 2015, A\&A, 576, A10

Civano F. et al., 2016, ApJ, 819, 62

Coil A. L., Newman J. A., Cooper M. C., Davis M., Faber S. M., Koo D. C., Willmer C. N. A., 2006, ApJ, 644, 671

Coil A. L., Mendez A. J., Eisenstein D. J., Moustakas J., 2017, ApJ, 838, 87

Conselice C. J., 2014, ARA\&A, 52, 291

Cooper M. C. et al., 2006, MNRAS, 370, 198

Darvish B., Sobral D., Mobasher B., Scoville N. Z., Best P., Sales L. V., Smail I., 2014, ApJ, 796, 51

Darvish B., Mobasher B., Sobral D., Scoville N., Aragon-Calvo M., 2015, ApJ, 805, 121

Darvish B., Mobasher B., Sobral D., Rettura A., Scoville N., Faisst A., Capak P., 2016, ApJ, 825, 113

Darvish B., Mobasher B., Martin D. C., Sobral D., Scoville N., Stroe A., Hemmati S., Kartaltepe J., 2017, ApJ, 837, 16

Davidzon I. et al., 2017, A\&A, 605, A70

Davis S. W., Laor A., 2011, ApJ, 728, 98

De Lucia G., Springel V., White S. D. M., Croton D., Kauffmann G., 2006 MNRAS, 366, 499

Dekel A. et al., 2009, Nature, 457, 451

Delvecchio I. et al., 2017, A\&A, 602, A3

Desjacques V., Jeong D., Schmidt F., 2016, Phys. Rep., 733, 1

Digby-North J. A. et al., 2010, MNRAS, 407, 846

Draine B. T., Li A., 2007, ApJ, 657, 810

Dressler A., 1980, ApJ, 236, 351

Ebeling H., Stephenson L. N., Edge A. C., 2014, ApJ, 781, L40

Ehlert S. et al., 2014, MNRAS, 437, 1942

Elbaz D. et al., 2007, A\&A, 468, 33

Farouki R., Shapiro S. L., 1981, ApJ, 243, 32

Finoguenov A. et al., 2007, ApJS, 172, 182

Foreman-Mackey D., Hogg D. W., Lang D., Goodman J., 2013, PASP, 125, 306

Fragos T. et al., 2013, ApJ, 764, 41

Georgakakis A., 2018, Athena Nuggets, 24

Georgakakis A., Gerke B. F., Nandra K., Laird E. S., Coil A. L., Cooper M. C., Newman J. A., 2008, MNRAS, 391, 183

Georgakakis A. et al., 2014, MNRAS, 443, 3327

Georgakakis A., Aird J., Schulze A., Dwelly T., Salvato M., Nandra K., Merloni A., Schneider D. P., 2017, MNRAS, 471, 1976

George M. R. et al., 2011, ApJ, 742, 125

Grogin N. A. et al., 2011, ApJS, 197, 35

Gunn J. E., Gott J. R., III, 1972, ApJ, 176, 1

Haggard D., Green P. J., Anderson S. F., Constantin A., Aldcroft T. L., Kim

D.-W., Barkhouse W. A., 2010, ApJ, 723, 1447

Hasinger G. et al., 2018, ApJ, 858, 77

Hickox R. C. et al., 2009, ApJ, 696, 891 
Hickox R. C., Mullaney J. R., Alexander D. M., Chen C.-T. J., Civano F. M., Goulding A. D., Hainline K. N., 2014, ApJ, 782, 9

Hopkins P. F., Hernquist L., Cox T. J., Di Matteo T., Robertson B., Springel V., 2006, ApJS, 163, 1

Hopkins P. F., Richards G. T., Hernquist L., 2007, ApJ, 654, 731

Hsu L.-T. et al., 2014, ApJ, 796, 60

Ilbert O. et al., 2009, ApJ, 690, 1236

Ilbert O. et al., 2010, ApJ, 709, 644

Ilbert O. et al., 2013, A\&A, 556, A55

Johnson R. A., Wichern D. W., 2002, Applied Multivariate Statistical Analysis. Vol. 5, Prentice Hall, Upper Saddle River, NJ

Karhunen K., Kotilainen J. K., Falomo R., Bettoni D., 2014, MNRAS, 441, 1802

Kauffmann G., White S. D. M., Heckman T. M., Ménard B., Brinchmann J., Charlot S., Tremonti C., Brinkmann J., 2004, MNRAS, 353, 713

Kereš D., Katz N., Weinberg D. H., Davé R., 2005, MNRAS, 363, 2

Kim S., 2015, Commun. Stat. Appl. Methods, 22, 665

King A., Pounds K., 2015, ARA\&A, 53, 115

Knobel C. et al., 2009, ApJ, 697, 1842

Knobel C. et al., 2012, ApJ, 753, 121

Koekemoer A. M. et al., 2011, ApJS, 197, 36

Kormendy J., Ho L. C., 2013, ARA\&A, 51, 511

Koulouridis E. et al., 2014, A\&A, 567, A83

Kravtsov A. V., Borgani S., 2012, ARA\&A, 50, 353

LaMassa S. M. et al., 2013, MNRAS, 436, 3581

Laigle C. et al., 2016, ApJS, 224, 24

Laigle C. et al., 2018, MNRAS, 474, 5437

Leauthaud A., et al., 2015, MNRAS, 446, 1874

Lehmer B. D. et al., 2009, ApJ, 691, 687

Lehmer B. D. et al., 2013, ApJ, 765, 87

Lehmer B. D. et al., 2016, ApJ, 825, 7

Li C., Kauffmann G., Wang L., White S. D. M., Heckman T. M., Jing Y. P., 2006, MNRAS, 373, 457

Lietzen H. et al., 2009, A\&A, 501, 145

Lilly S. J. et al., 2009, ApJS, 184, 218

Lin L. et al., 2010, ApJ, 718, 1158

Liu T. et al., 2017, ApJS, 232, 8

Luo B. et al., 2010, ApJS, 187, 560

Luo B. et al., 2017, ApJS, 228, 2

Lusso E. et al., 2012, MNRAS, 425, 623

Macuga M. et al., 2018, preprint (arXiv:1805.06569)

Madau P., Dickinson M., 2014, ARA\&A, 52, 415

Malavasi N., Pozzetti L., Cucciati O., Bardelli S., Cimatti A., 2016, A\&A, 585, A116

Marchesi S. et al., 2016a, ApJ, 817, 34

Marchesi S. et al., 2016b, ApJ, 830, 100

Marconi A., Risaliti G., Gilli R., Hunt L. K., Maiolino R., Salvati M., 2004, MNRAS, 351, 169

Martini P., Schneider D. P., 2003, ApJ, 597, L109

Martini P., Sivakoff G. R., Mulchaey J. S., 2009, ApJ, 701, 66

Martini P. et al., 2013, ApJ, 768, 1

McCracken H. J. et al., 2012, A\&A, 544, A156

Mendez A. J., et al., 2016, ApJ, 821, 55

Meneux B. et al., 2009, A\&A, 505, 463

Merloni A. et al., 2012, preprint (arXiv:1209.3114)

Miyaji T., Krumpe M., Coil A. L., Aceves H., 2011, ApJ, 726, 83

Moore B., Lake G., Katz N., 1998, ApJ, 495, 139

Mundy C. J., Conselice C. J., Duncan K. J., Almaini O., Häußler B., Hartley W. G., 2017, MNRAS, 470, 3507

Murtaugh P. A., 2014, Ecology, 95, 611

Noll S., Burgarella D., Giovannoli E., Buat V., Marcillac D., Muñoz-Mateos J. C., 2009, A\&A, 507, 1793

Novak G. S., Ostriker J. P., Ciotti L., 2011, ApJ, 737, 26

Overzier R. A., 2016, A\&AR, 24, 14

Peng Y.-j. et al., 2010, ApJ, 721, 193

Pierre M. et al., 2016, A\&A, 592, A1

Poggianti B. M. et al., 2016, AJ, 151, 78

Poggianti B. M. et al., 2017, Nature, 548, 304
Powell M. C. et al., 2018, ApJ, 858, 110

Reddick R. M., Wechsler R. H., Tinker J. L., Behroozi P. S., 2013, ApJ, 771, 30

Richardson J., Chatterjee S., Zheng Z., Myers A. D., Hickox R., 2013, ApJ, 774,143

Sartori L. F., Schawinski K., Trakhtenbrot B., Caplar N., Treister E., Koss M. J., Megan Urry C., Zhang C., 2018, MNRAS, 476, L34

Scoville N. et al., 2007, ApJS, 172, 150

Scoville N. et al., 2013, ApJS, 206, 3

Serber W., Bahcall N., Ménard B., Richards G., 2006, ApJ, 643, 68

Serra P., Amblard A., Temi P., Burgarella D., Giovannoli E., Buat V., Noll S., Im S., 2011, ApJ, 740, 22

Silverman J. D. et al., 2009, ApJ, 695, 171

Skibba R. A. et al., 2014, ApJ, 784, 128

Somerville R. S., Davé R., 2015, ARA\&A, 53, 51

Springel V. et al., 2005, Nature, 435, 629

Steffen A. T., Strateva I., Brandt W. N., Alexander D. M., Koekemoer A. M., Lehmer B. D., Schneider D. P., Vignali C., 2006, AJ, 131, 2826

Steidel C. C., Adelberger K. L., Dickinson M., Giavalisco M., Pettini M., Kellogg M., 1998, ApJ, 492, 428

Strand N. E., Brunner R. J., Myers A. D., 2008, ApJ, 688, 180

Topping M. W., Shapley A. E., Steidel C. C., 2016, ApJ, 824, L11

Umehata H. et al., 2015, ApJ, 815, L8

Vasudevan R. V., Fabian A. C., 2007, MNRAS, 381, 1235

Vito F. et al., 2014, MNRAS, 441, 1059

Vito F. et al., 2016, MNRAS, 463, 348

Wang T. et al., 2017, A\&A, 601, A63

Williams R. J., Quadri R. F., Franx M., van Dokkum P., Labbé I., 2009, ApJ, 691,1879

Xue Y. Q., 2017, New Astron. Rev., 79, 59

Xue Y. Q. et al., 2010, ApJ, 720, 368

Xue Y. Q., Luo B., Brandt W. N., Alexander D. M., Bauer F. E., Lehmer B. D., Yang G., 2016, ApJS, 224, 15

Yang G. et al., 2014, ApJS, 215, 27

Yang G. et al., 2016, ApJ, 831, 145

Yang G. et al., 2017, ApJ, 842, 72

Yang G. et al., 2018, MNRAS, 475, 1887

\section{SUPPORTING INFORMATION}

Supplementary data are available at MNRAS online.

Table 2. Source catalogue.

Please note: Oxford University Press is not responsible for the content or functionality of any supporting materials supplied by the authors. Any queries (other than missing material) should be directed to the corresponding author for the article.

\section{APPENDIX: EXPLANATION OF ENVIRONMENT MEASUREMENTS}

Our environment estimation in Section 2.3 is $2 \mathrm{D}$ in nature (projected over $\approx 80-200 \mathrm{Mpc}$ along the line of sight (LOS); see Fig. A1). Admittedly, the $2 \mathrm{D}$ environment measurements have limitations and cannot fully recover the entire 3D environment. However, through intensive tests on simulated data, studies have found that the $2 \mathrm{D}$ environment estimates can reliably trace the intrinsic 3D environments. For example, Scoville et al. (2013) found that the projected $2 \mathrm{D}$ densities are monotonically related to the true $3 \mathrm{D}$ volume densities with a power-law slope of $\approx 0.67$. Laigle et al. (2018) found that the $2 \mathrm{D}$ measured filaments robustly match their $3 \mathrm{D}$ counterparts. These strong 2D-3D correlations result from the fact that, in the projection, the chance for different structures to overlap is low. The low overlapping probability is caused by the facts that most ( $\gtrsim 80$ per cent) of the 3D space is the field environment in 

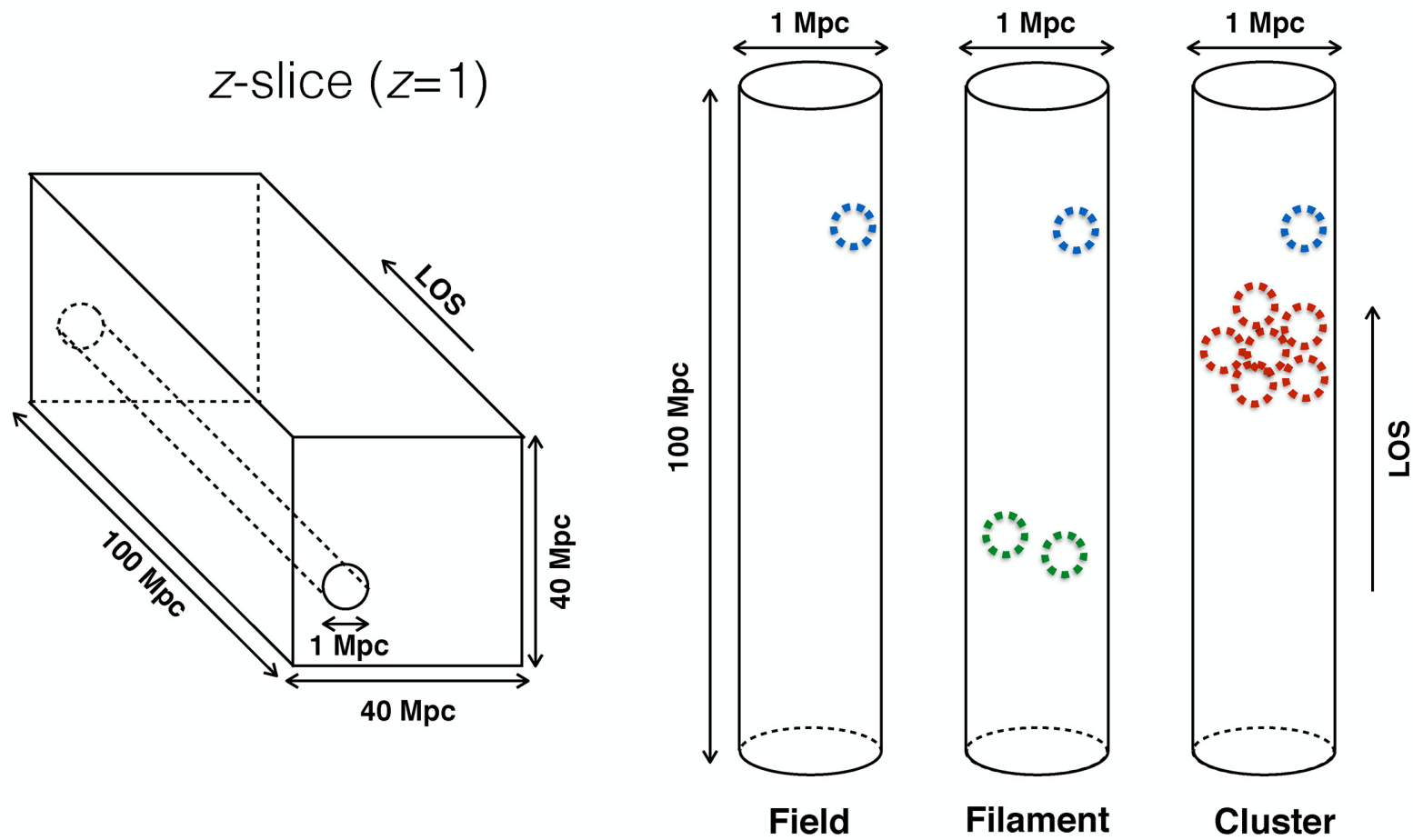

Figure A1. Schematic plot for overdensity measurements (not drawn to scale). The left-hand panel shows the redshift slice at $z=1$. The $z$-slice contains galaxies projected along the LOS over $\approx 100 \mathrm{Mpc}$. Our surface density is measured in cylinders with radii $\approx 0.5 \mathrm{Mpc}$ as marked. The right-hand panel illustrates three typical cylinders for the field, filament, and cluster environments, respectively. The dashed circles denote galaxies inside the cylinders. Blue, green, and red colours indicate galaxies associated with 3D field, filament, and cluster environments, respectively. The numbers of galaxies plotted reflect the typical galaxy numbers in our measurements at $z \approx 1$.

$\Lambda$ cold dark matter simulations (e.g. Aragón-Calvo, van de Weygaert \& Jones 2010; Cautun et al. 2014) and that low-mass haloes might not host galaxies and are thus unobservable (e.g. Desjacques, Jeong \& Schmidt 2016, and references therein). Assuming Poisson fluctuations, we estimate the chance for two (or more) overlapping filaments along an LOS is $\lesssim 3$ per cent, based on the fact that the filament environment covers $\lesssim 30$ per cent of the total area (see Figs 2 and 3). Although a rigorous quantitative demonstration on simulated data is beyond the scope of this work, we qualitatively explain our 2D environment measurements in a straightforward way below.

Taking our $z$-slice at $z=1$ as an example (Fig. 2), we show the scheme of our environment measurements in Fig. A1. ${ }^{7}$ Our surface-density field is measured within a $2 \mathrm{D}$ circle with a radius of $\approx 0.5 \mathrm{Mpc}$, projected from a 3D cylinder of length $\approx 100 \mathrm{Mpc}$ (Fig. A1, left). Fig. A1 (right) shows typical field, filament, and cluster environments. The numbers of galaxies plotted reflect the typical galaxy numbers in our measurements for different environments at $z \approx 1$. For the field environment, our surface density is averaged over the whole cylinder with a volume of $\pi \times 0.5^{2} \times 100 \mathrm{Mpc}^{3}$. This relatively large volume is necessary to include $\gtrsim 1$ galaxies.

\footnotetext{
${ }^{7}$ Technically, the measurements are more complicated (see Section 2.3), the schematic plot here is just for demonstration purposes.
}

For the filament environment, the density enhancement is mainly due to the 3D dense region with scale similar to the filament 'thickness' ( $\lesssim 1$ Mpc scales; see Figs 2 and 3). The situation for the cluster environment is similar to that for filament environment.

Admittedly, environment mis-classification might happen in some cases. For example, a filament, when it aligns with the LOS, might be mis-classified as a cluster. However, this situation should be rare because filaments are often not straight and have curved shapes (see Figs 2 and 3). Also, galaxies in the cluster environment generally have significantly lower SFR than those in the filament environment at $z \lesssim 1$ (e.g. Darvish et al. 2017; Fig. 16). This physical phenomenon would not be observed if our classified cluster population is heavily polluted by an intrinsic filament population.

Due the existence of various projection effects, any quantitative correlation with $2 \mathrm{D}$ environment should not be literally interpreted as a quantitative correlation with the intrinsic 3D environment. For example, a quantity ' $A$ ' is found to be positively correlated with $2 \mathrm{D}$ overdensity with a power-law index of $\alpha$. We can only conclude qualitatively that $A$ is positively related to $3 \mathrm{D}$ overdensity, but not quantitatively that the relation between $A$ and $3 \mathrm{D}$ overdensity is also a power law with an index of $\alpha$.

This paper has been typeset from a $\mathrm{TE}_{\mathrm{E}} \mathrm{X} / \mathrm{L} \mathrm{T} \mathrm{E}$ file prepared by the author. 